\title{
Differential Geometry of Identity Maps: A Survey
}

\section{Bang-Yen Chen}

Department of Mathematics, Michigan State University, 619 Red Cedar Road, East Lansing, MI 48824-1027, USA; chenb@msu.edu

Received: 02 July 2020; Accepted: 28 July 2020; Published: 2 August 2020

Abstract: An identity map $i d_{M}: M \rightarrow M$ is a bijective map from a manifold $M$ onto itself which carries each point of $M$ return to the same point. To study the differential geometry of an identity map $i d_{M}: M \rightarrow M$, we usually assume that the domain $M$ and the range $M$ admit metrics $g$ and $g^{\prime}$, respectively. The main purpose of this paper is to provide a comprehensive survey on the differential geometry of identity maps from various differential geometric points of view.

Keywords: identity map; harmonic map; biharmonic map; Gauss map; Laplace map; harmonic metrics; Walker manifold; Gödel-type space time; index; nullity

MSC: 53C05; 53C40; 53A40; 53C15

\section{Contents}

1 Introduction $\quad 2$

2 Pseudo-Riemannian Manifolds and Submanifolds 3

2.1 Basics on Pseudo-Riemannian Manifolds . . . . . . . . . . . . . . . . . . . . . 4

2.2 Bianchi Identities . . . . . . . . . . . . . . . . . . . . . . . 5

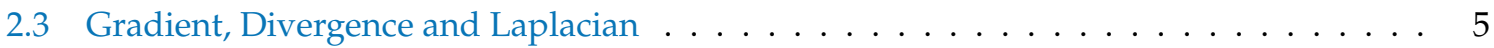

2.4 Basics on Submanifolds . . . . . . . . . . . . . . . . . . . 6

2.5 Submanifolds of Finite Type . . . . . . . . . . . . . . . . . . . . . 6

2.6 Linearly Independent and Orthogonal Immersions . . . . . . . . . . . . . . . . . 7

3 Harmonic Maps $\quad \mathbf{8}$

3.1 Harmonic Map and Tension Field . . . . . . . . . . . . . . . . . . . . . . . . . . . . . . . . .

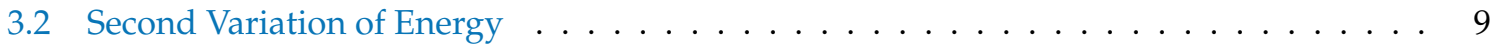

3.3 Stability, Index and Nullity . . . . . . . . . . . . . . . . . . . . 9

4 Identity Maps, Harmonic Metrics and Harmonic Tensors 10

4.1 Relative Harmonic Metrics . . . . . . . . . . . . . . . . . . . . . . . . . . . . 10

4.2 Space of Harmonic Tensors . . . . . . . . . . . . . . . . . . . . . 11

4.3 Links between Geodesic Vector Fields and Harmonic Tensors ． . . . . . . . . . . . 12

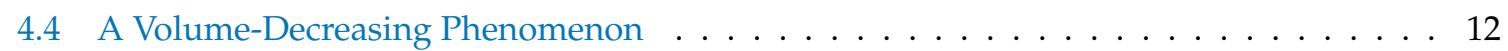

4.5 Identity Maps on Warped Products . . . . . . . . . . . . . . . . . . . . . . 13

4.6 Identity Maps on Kaehler and Hyper-Kaehler Manifolds . . . . . . . . . . . . . . . . 13

5 Walker Metrics and Identity Maps $\quad 14$

5.1 Walker Manifolds . . . . . . . . . . . . . . . . . . . . . . . . . . . . . 14

5.2 Equivalence Classes of Walker 4-Manifolds . . . . . . . . . . . . . . . . . . . . . . . . . . . . . . . . . . . . . . . .

5.3 Relative Harmonic Metrics on Walker 4-Manifolds . . . . . . . . . . . . . . . . . . . 14 
6 Index and Nullity of Identity Maps $\quad 15$

6.1 Nullity of Identity Maps . . . . . . . . . . . . . . . . . . . . . . . . . . . . . . . . . . . . . . . . . . . . .

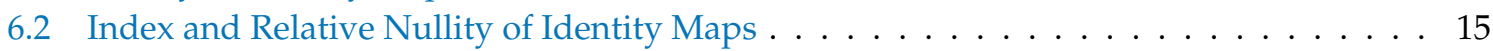

6.3 Index and Nullity of Spheres and Flat Torus . . . . . . . . . . . . . . . . . . . 16

6.4 Index and Nullity of Product Maps . . . . . . . . . . . . . . . . . . . 16

7 Identity Maps on Compact Symmetric Spaces $\quad \mathbf{1 7}$

7.1 Stability of Compact Symmetric Spaces . . . . . . . . . . . . . . . . . . . . 17

7.2 Instability of Compact Symmetric Spaces . . . . . . . . . . . . . . . . . . 18

8 Identity Maps of Other Spaces $\quad 18$

8.1 Identity Maps on Homogeneous Space $(S U(3) / T(k, \ell), g) \ldots \ldots$

8.2 Stability of Spheres with Deformed Metrics . . . . . . . . . . . . . . . . . . . . . . . . . . . . . . . . . . . .

8.3 Identity Maps and Riemannian Submersions . . . . . . . . . . . . . . . . . . . 19

8.4 Identity Maps on Generalized Sasakian Space Forms ～. . . . . . . . . . . . . . . . . 20

9 Biharmonic Identity Maps $\quad 21$

9.1 Biharmonic Maps and Biharmonic Submanifolds . . . . . . . . . . . . . . . . . . . . . 21

9.2 Biharmonic Maps and Relative Biharmonic Metrics . . . . . . . . . . . . . . . . . 21

9.3 Obstructions to Biharmonic Metrics on Einstein Manifolds . . . . . . . . . . . . . . . 23

9.4 A Biharmonicity Reduction Theorem For Submersions . . . . . . . . . . . . . . . 23

10 Gauss and Identity Maps $\quad 23$

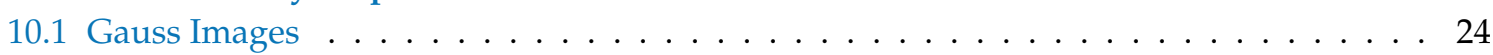

10.2 Geometry of Gauss Transformations . . . . . . . . . . . . . . . . . . . . 24

11 Laplace and Identity Maps $\quad 25$

11.1 Submanifolds with Homothetic Laplace Transformations . . . . . . . . . . . . . . . . 25

11.2 Geometry of Conformal or Harmonic Laplace Transformations . . . . . . . . . . . . . . . 26

11.3 Geometry of Laplace-Gauss Identity Maps . . . . . . . . . . . . . . . . . . . . . 26

12 Identity Maps of Tangent Bundles $\quad 27$

12.1 Tangent Bundles . . . . . . . . . . . . . . . . . . . . . . . . 27 27

12.2 Identity Maps of Tangent Bundles with Lift-Complete Metrics . . . . . . . . . . . . . . 28

12.3 Identity Maps of Tangent Bundles with g-Natural Metrics . . . . . . . . . . . . . . . . . 28

12.4 Harmonic Metrics on Non-Reductive Homogeneous Manifolds . . . . . . . . . . . . . 29

12.5 Identity Maps of Tangent Bundles of Walker 4-Spaces . . . . . . . . . . . . . . . . . . . 29

12.6 Identity Maps of Gödel-Type Spacetimes . . . . . . . . . . . . . . . . . . . . . . . . 30

$\begin{array}{lr}\text { References } & 30\end{array}$

\section{Introduction}

An identity map $i d_{M}: M \rightarrow M$ from a differentiable manifold $M$ into itself, also known as an identity transformation, is a map that always returns to the same point that was used in as its argument. In other words, the identity map $i d_{M}$ on a manifold $M$ is defined to be the map with domain and range $M$ which satisfies

$$
i d_{M}(p)=p \text { for any } p \in M
$$

Obviously, the identity map $i d_{M}: M \rightarrow M$ is the simplest map which is both continuous and bijective. 
The main purpose of studying the differential geometry of identity maps is to investigate the following problem:

\section{"To what extent does the identity map of a manifold determine the geometry of the manifold?"}

In general, to study the differential geometry of an identity map $i d_{M}: M \rightarrow M$, we shall assume that the domain $M$ and the range $M$ admit two geometric structures of the same kind. In [1], Nagano proved that the identity map $i d_{M}:(M, g) \rightarrow(M, g)$ of a compact Riemannian manifold $(M, g)$ onto itself is a stable map if and only if the diagonal map in $M \times M$ is stable as a minimal submanifold. Further, the author and Nagano proved in [2] that a Riemannian manifold $(M, g)$ admits a geodesic vector field $v$ if and only if the identity map $i d_{M}:(M, g) \rightarrow\left(M, \mathcal{L}_{v} g\right)$ is a harmonic map, where $\mathcal{L}_{v}$ denotes the Lie derivative with respect to $v$. For two linear connections $\Gamma$ and $\Gamma^{\prime}$ on a manifold $M$, the identity map $i d_{M}:(M, \Gamma) \rightarrow\left(M, \Gamma^{\prime}\right)$ is called affine if the identity map $i d_{M}$ carries every parallel vector field along each curve $\gamma$ of $(M, \Gamma)$ into a parallel vector field along $\gamma$ in $\left(M, \Gamma^{\prime}\right)$. It is known from ([3] page 225) that the identity map $i d_{M}:(M, \Gamma) \rightarrow\left(M, \Gamma^{\prime}\right)$ is affine if and only if it carries each geodesic of $(M, \Gamma)$ into a geodesic of $\left(M, \Gamma^{\prime}\right)$.

To study the conformal geometry of the identity map on a manifold $M$, we assume that domain $M$ and range $M$ equipped with metrics $g$ and $g^{\prime}$, respectively. The identity map $i d_{M}:(M, g) \rightarrow\left(M, g^{\prime}\right)$ is called a conformal change of metric if $g^{\prime}=\rho^{2} g$ for some positive function $\rho$. It is well-known that, in the case $\operatorname{dim} M \geq 4$, if the identity map $i d_{M}:(M, g) \rightarrow\left(M, g^{\prime}\right)$ is a conformal change of metric, then $(M, g)$ and $\left(M, g^{\prime}\right)$ have the same Weyl tensor (cf. [4]); and in the case $\operatorname{dim} M=3,(M, g)$ and $\left(M, g^{\prime}\right)$ have the same Cotton tensor (cf. e.g., [5,6]).

The main purpose of this paper is to provide a survey on known results about identity maps from various Riemannian geometric points of view.

This article is organized as follows. The basic materials on pseudo-Riemannian manifolds is given in the second section. Section 3 provides a brief introduction on harmonic maps including the first and the second variations of Dirichlet energy functionals as well as the stability, index and nullities of harmonic maps. In Section 4, we discuss the relationship between identity maps and (relative) harmonic metrics, (relative) harmonic tensor and geodesic vector fields. We also present a volume-decreasing phenomenon for co-closed harmonic metrics. We mention a necessary and sufficient conditions for the warped product metric of two Einstein manifolds to be harmonic as well as identity maps on Kaehler and hyper-Kaehler manifolds. In Section 5, we present the result for a Walker metric (with neutral signature) on a Walker 4-manifold $W^{4}$ to be harmonic with respect to another Walker metric. In Section 6, we discuss index, relative nullity and Killing nullity of identity maps for several important Riemannian manifolds. In addition, results on stabilities and nullities of compact symmetric spaces are presented in this section. In Section 7, we discuss identity maps on compact symmetric spaces. In Section 8, we survey results on the stability of some other important spaces; including homogeneous spaces, spheres, flat tori, and their products, as well as the stability of generalized Sasakian space forms. In Section 9, we present some known results on biharmonic identity maps; including biharmonic metrics, obstructions to the existence of biharmonic metrics on Einstein manifolds, and biharmonicity of Riemannian submersions. In Section 10, we provide the relationship between Gauss maps and identity maps, mainly for surfaces. In Section 11, we present results on Laplace maps and the related identity maps proved in the book [7]. In the last section, we present results on the identity maps of tangent bundles of manifolds equipped with Sasakian, lift-complete, or $g$-natural metrics. In addition, we discuss the identity maps on the tangent bundles of Walker 4-manifolds and of Gödel-type spacetimes.

\section{Pseudo-Riemannian Manifolds and Submanifolds}

We follow the notations given in [6,8-10]. 


\subsection{Basics on Pseudo-Riemannian Manifolds}

Consider a smooth $n$-manifold $M$ covered by a system of coordinate neighborhoods $\left(U,\left\{x^{i}\right\}\right)$, where $U$ is a neighborhood and the $\left\{x^{i}\right\}$ denote local coordinates on $U$, where the indices $i, j, k, \ell, \ldots$ take on values in the range $1,2, \ldots, n$. Then a Riemannian (or pseudo-Riemannian) metric $g$ on $M$ can be expressed as

$$
g=g_{i j} d x^{i} d x^{j}
$$

where we have applied the Einstein convention, i.e., repeated indices, with one upper index and one lower index, denoted summation over its range.

In the following, we denote by $\partial_{i}=\frac{\partial}{\partial x_{i}}$ the basis vectors on the coordinate neighborhood $\left\{U,\left(x^{1}, \ldots, x^{n}\right)\right\}$. Let $X$ and $Y$ be two vector fields on $M$. We then have

$$
X=X^{h} \partial_{h}, \quad Y=Y^{h} \partial_{h}
$$

where $X^{h}$ and $Y^{h}$ are the local components of the vector fields $X$ and $Y$, respectively. With respect to the natural frame $\partial_{h}$, we have

$$
g_{i j}=g\left(\partial_{i}, \partial_{j}\right)
$$

which are the local components of the metric tensor field $g$. The Christoffel symbols of the Riemannian manifold $(M, g)$ are given by

$$
\Gamma_{i j}^{k}=\frac{g^{k \ell}}{2}\left(\partial_{j} g_{i \ell}+\partial_{i} g_{j \ell}-\partial_{\ell} g_{i j}\right),
$$

where $\left(g^{k \ell}\right)$ denotes the inverse matrix of $\left(g_{i j}\right)$. Thus, we have $g_{i j} g^{j k}=\delta_{i}^{k}$, where $\delta_{i}^{k}=1$ or 0 according to $i=k$ or $i \neq k$.

Let $\nabla$ denote the Levi-Civita connection of $(M, g)$. Then for vector fields $X, Y$ on $M$ the covariant derivative $\nabla_{X} Y$ has local components

$$
X^{j} \nabla_{j} Y^{h}=X^{j}\left(\partial_{j} Y^{h}+\Gamma_{j i}^{h} Y^{i}\right)
$$

The covariant derivative of a tensor $T_{i j}$ of type $(0,2)$ is defined by

$$
\nabla_{k} T_{i j}=\partial_{k} T_{i j}-\Gamma_{k i}^{h} T_{h j}-\Gamma_{k j}^{h} T_{h i}
$$

Let $X, Y$ and $Z$ be three vector fields in $M$. Then

$$
\nabla_{X} \nabla_{Y} Z-\nabla_{Y} \nabla_{X} Z-\nabla_{[X, Y]} Z
$$

defines a tensor field $K$ of type $(1,3)$. If we put

$$
K(X, Y) Z=\nabla_{X} \nabla_{Y} Z-\nabla_{Y} \nabla_{X} Z-\nabla_{[X, Y]} Z,
$$

then $K(X, Y)$ is a tensor field of type $(1,1)$ which is linear both in $X$ and $Y$. In terms of local components, (6) can be written as

$$
K_{k j i}^{h} Z^{i}=\nabla_{k} \nabla_{j} Z^{h}-\nabla_{j} \nabla_{k} Z^{h}
$$

where

$$
K_{k j i}^{h}=\partial_{k} \Gamma_{j i}^{h}-\partial_{j} \Gamma_{k i}^{h}+\Gamma_{k \ell}^{h} \Gamma_{j i}^{\ell}-\Gamma_{j \ell}^{h} \Gamma_{k i}^{\ell}
$$

are the local components of the curvature tensor $K$. 
The Ricci tensor Ric of $(M, g)$ is the $(0,2)$-tensor with local components given by

$$
\operatorname{Ric}_{i j}=K_{k i j}{ }^{k}=g^{k h} K_{k j i h} .
$$

The scalar curvature $r$ is given by

$$
r=g^{i j} \operatorname{Ric}_{i j} .
$$

Let $\mathcal{T}_{s}^{r}$ denote the space of all tensors of type $(r, s)$ on $(M, g)$. Following [2], we denote by $\delta$ the codifferential of $\mathcal{T}_{s}^{r}$ defined by

$$
(\delta T)_{i_{1} \cdots i_{s}}^{j_{1} \cdots j_{r}}=-\nabla^{k} T_{k i_{1} \cdots i_{s}}^{j_{1} \cdots j_{r}}
$$

Then $\delta: \mathcal{T}_{s+1}^{r} \rightarrow \mathcal{T}_{s}^{r}$. As in [2], a tensor $T$ is called co-closed if it satisfies $\delta T=0$.

\subsection{Bianchi Identities}

The curvature tensor $K$ satisfies the first Bianchi identity

$$
K(X, Y) Z+K(Y, Z) X+K(Z, X) Y=0
$$

or in local components

$$
K_{k j i h}+K_{j i k h}+K_{i k j h}=0 .
$$

The curvature tensor $K$ also satisfies the second Bianchi identity

$$
\left(\nabla_{X} K\right)(Y, Z)+\left(\nabla_{Y} K\right)(Z, X)+\left(\nabla_{Z} K\right)(X, Y)=0,
$$

or in local components

$$
\nabla_{\ell} K_{k j i h}+\nabla_{k} K_{j \ell i h}+\nabla_{j} K_{\ell k i h}=0 .
$$

\subsection{Gradient, Divergence and Laplacian}

Assume $(M, g)$ is a pseudo-Riemannian manifold and $f$ is a smooth function on $M$. Then the gradient of $f$, denote by $\nabla f$ (or by grad $f$ ), is the vector field dual to the differential $d f$. In other word, $\nabla f$ is defined by

$$
\langle\nabla f, X\rangle=d f(X)=X f \quad \forall X \in T(M) .
$$

In terms of a local coordinate system $\left\{x^{i}\right\}$ of $M$, the divergence of a vector field $X=X^{j} \partial_{j}$, denoted by $\operatorname{div} X$, is given by

$$
\operatorname{div} X=\sum_{j=1}^{n}\left\{\frac{\partial X^{j}}{\partial x^{j}}+\sum_{k=1}^{n} \Gamma_{j k}^{j} X^{k}\right\} .
$$

The Laplacian of $f$, denoted by $\Delta f$, is defined by $\Delta f=-\operatorname{div}(\nabla f)$, we have

$$
\Delta f=-\sum_{i, j=1}^{n} g^{i j}\left\{\frac{\partial^{2} f}{\partial x^{i} \partial x^{j}}-\sum_{k=1}^{n} \Gamma_{i j}^{k} \frac{\partial f}{\partial x^{k}}\right\} .
$$




\subsection{Basics on Submanifolds}

Assume that $\phi:(M, g) \rightarrow(N, h)$ is an isometric immersion of a pseudo-Riemannian manifold into another. Denote by $\nabla$ and $\nabla^{N}$ the Levi Civita connections on $M$ and $N$, respectively. The formulas of Gauss and Weingarten are given respectively by

$$
\begin{aligned}
& \nabla_{X}^{N} Y=\nabla_{X} Y+B(X, Y), \\
& \nabla_{X}^{N} \xi=-A_{\xi} X+D_{X} \xi
\end{aligned}
$$

for tangent vector fields $X, Y$ and a normal vector field $\xi$, where $B, A$ and $D$ are the second fundamental form, the shape operator and the normal connection.

For each $\xi \in T_{p}^{\perp} M$, the shape operator $A_{\xi}$ is a symmetric endomorphism of the tangent space $T_{p} M$ at $p \in M$.

The shape operator and the second fundamental form are related by

$$
\langle B(X, Y), \xi\rangle=\left\langle A_{\xi} X, Y\right\rangle
$$

for $X, Y$ tangent to $M$ and $\xi$ normal to $M$. The mean curvature vector is defined by $H=\frac{1}{2}$ trace $h$.

For a vector $\widetilde{X} \in T_{p} N, p \in M$, we denote by $\widetilde{X}^{\top}$ and $\widetilde{X}^{\perp}$ the tangential and the normal components of $\widetilde{X}$, respectively. The equations of Gauss, Codazzi and Ricci are given respectively by

$$
\begin{aligned}
& \left(R^{N}(X, Y) Z\right)^{T}=R(X, Y) Z+A_{B(X, Z)} Y-A_{B(Y, Z)} X \\
& \left(R^{N}(X, Y) Z\right)^{\perp}=\left(\bar{\nabla}_{X} B\right)(Y, Z)-\left(\bar{\nabla}_{Y} B\right)(X, Z) \\
& \left(R^{N}(X, Y) \xi\right)^{\perp}=h\left(A_{\xi} X, Y\right)-h\left(X, A_{\xi} Y\right)+R^{D}(X, Y) \xi
\end{aligned}
$$

for vector fields $X, Y, Z$ tangent to $M, \xi$ normal to $M$, and $\bar{\nabla} B$ is given by

$$
\left(\bar{\nabla}_{X} B\right)(Y, Z)=D_{X} B(Y, Z)-h\left(\nabla_{X} Y, Z\right)-B\left(Y, \nabla_{X} Z\right),
$$

and $R^{D}$ is the curvature tensor associated to the normal connection $D$.

The submanifold $M$ is called totally geodesic if $B=0$ holds identically; and parallel if $\bar{\nabla} B=0$ identically. The submanifold is said to have parallel mean curvature vector if $D H=0$ identically (cf. [11] for a detailed survey on submanifolds with parallel mean curvature vector).

\subsection{Submanifolds of Finite Type}

The theory of finite type submanifolds was initiated in [12-14]. The first results on finite type submanifolds were collected in the book [15]. Since that time, the subject has developed rapidly. A detailed survey on results up to 1996 was given in author's report [16]. The most recent comprehensive survey on this subject was given in author's book [9] (see also [17-19]).

A submanifold $M$ of $\mathbb{E}^{m}$ is said to be of finite type if its immersion $\phi$ is a finite sum of eigenmaps of the Laplacian $\Delta$, i.e.,

$$
\phi=c_{0}+\phi_{1}+\cdots+\phi_{k}
$$

where $c_{0}$ is a constant map and $\phi_{i}, \ldots, \phi_{k}$ are non-constant maps satisfying

$$
\Delta \phi_{i}=\lambda_{t_{i}} \phi_{i}, \quad \lambda_{t_{i}} \in \mathbb{R}, \quad i=1, \ldots, k .
$$

If the eigenvalues $\lambda_{t_{1}}, \ldots, \lambda_{t_{k}}$ of $\Delta$ in (27) are distinct, then $M$ (or the immersion $\phi$ ) is said to be $k$-type. In particular, if one of $\lambda_{t_{1}}, \ldots, \lambda_{t_{k}}$ is zero, then $M$ is said to be of null $k$-type.

Analogously, a smooth map $\psi: M \rightarrow \mathbb{E}^{m}$ of a Riemannian manifold $M$ is called a finite type map if $\psi$ is a finite sum of $\mathbb{E}^{m}$-valued eigenmaps of $\Delta$. In the same way, we also have the notion of $k$-type 
maps and null $k$-type maps. An isometric immersion (or a map) of a Riemannian manifold into $\mathbb{E}^{m}$ is said to be of infinite type if it is not of finite type.

For each $\phi_{i}$ in the spectral decomposition (27) of $\phi$, we put

$$
E_{i}=\operatorname{Span}\left\{\phi_{i}(p): p \in M\right\}
$$

Then each $E_{i}$ is a linear subspace of $\mathbb{E}^{m}$.

\subsection{Linearly Independent and Orthogonal Immersions}

Consider a $k$-type isometric immersion $\phi: M \rightarrow \mathbb{E}^{m}$ whose spectral decomposition is given by (26). Then $\phi$ is said to be linearly independent if the subspaces $E_{1}, \ldots, E_{k}$ defined by (28) are linearly independent, i.e., the dimension of the subspace spanned by all vectors in $E_{1} \cup \ldots \cup E_{k}$ is equal to $\operatorname{dim}\left(E_{1}\right)+\ldots+\operatorname{dim}\left(E_{k}\right)$. The immersion $\phi$ is said to be orthogonal if the subspaces $E_{1}, \ldots, E_{k}$ are mutually orthogonal in $\mathbb{E}^{m}$ (see $[20,21]$ ).

If we choose a Euclidean coordinate system $\left\{u_{i}\right\}$ on $\mathbb{E}^{m}$ with $c$ as its origin, then the spectral decomposition (26) of $\phi$ reduces to

$$
\phi=\phi_{1}+\ldots+\phi_{k}, \Delta \phi_{i}=\lambda_{i} \phi_{i}, \lambda_{1}<\ldots<\lambda_{k} .
$$

For each $i \in\{1, \ldots, k\}$, we choose a basis $\left\{c_{i j}: j=1, \ldots, m_{i}\right\}$ of $E_{i}$, where $m_{i}$ denotes the dimension of $E_{i}$. Put $\ell=m_{1}+\ldots+m_{k}$ and let $E^{\ell}$ denote the subspace of $\mathbb{E}^{m}$ spanned by $E_{1}, \ldots, E_{k}$. If the immersion $\phi$ is linearly independent, then $\left\{c_{i j}: i=1, \ldots, k ; j=1, \ldots, m_{i}\right\}$ are $\ell$ linearly independent vectors in $E^{\ell}$. Further, we may choose the Euclidean coordinate system $\left\{u_{i}\right\}$ on $\mathbb{E}^{m}$ in such way that $E^{\ell}$ is defined by $u_{\ell+1}=\ldots=u_{m}=0$.

We regard each $c_{i j}$ as a column $\ell$-vector and put

$$
S=\left(c_{11}, \ldots, c_{1 m_{1}}, \ldots, c_{k 1}, \ldots, c_{k m_{k}}\right),
$$

then $S$ is a nonsingular $(\ell \times \ell)$-matrix. Let $D$ denote the diagonal $(\ell \times \ell)$-matrix

$$
D=\operatorname{Diag}(\overbrace{\lambda_{1}, \ldots, \lambda_{1}}^{m_{1} \text { times }}, \ldots, \overbrace{\lambda_{k}, \ldots, \lambda_{k}}^{m_{k} \text { times }})
$$

where $\lambda_{i}$ repeats $m_{i}$-times for $i=1, \ldots, k$. If we put $A=S D S^{-1}$, then we get $A c_{i j}=\lambda_{i} c_{i j}$ for any $i \in\{1, \ldots, k\}$ and $j \in\left\{1, \ldots, m_{i}\right\}$. Thus, we obtain

$$
\Delta \phi=A \phi
$$

for the immersion $\phi: M \rightarrow \mathbb{E}^{\ell}$ induced from $\phi: M \rightarrow \mathbb{E}^{m}$. We may regard the $(\ell \times \ell)$-matrix $A$ as an $(m \times m)$-matrix in a natural way (with zeros for each of the additional entries). For this $(m \times m)$-matrix $A$, we also have

$$
\Delta \phi=A \phi, \quad A=\left(a_{i j}\right)
$$

By using the $(m \times m)$-matrix $A$ in (30), the author introduced in [20] the notion of adjoint hyperquadric as follows.

Let $\phi: M \rightarrow \mathbb{E}^{m}$ be a non-minimal, linearly independent immersion whose spectral decomposition is given by (26). Let $\left\{u_{i}\right\}$ be a Euclidean coordinate system on $\mathbb{E}^{m}$ with $c$ as its origin. Let $A$ be the $(m \times m)$-matrix given in (30). Then, for a given point $p \in M$,

$$
\langle A u, u\rangle:=\sum_{i, j}^{m} a_{i j} u_{i} u_{j}=c_{p}, \quad\left(c_{p}=\langle A \phi, \phi\rangle(p)\right)
$$


defines a hyperquadric $Q_{p}$ in $\mathbb{E}^{m}$, called the adjoint hyperquadric of $\phi$ at $p$. In particular, if $\phi(M)$ lies in an adjoint hyperquadric $Q_{p}$ of $\phi$ for some point $p \in M$, then all of the adjoint hyperquadrics $\left\{Q_{p}\right.$ : $p \in M\}$ give the same adjoint hyperquadric which is denoted by $Q$, called the adjoint hyperquadric of the linearly independent immersion $\phi$.

\section{Harmonic Maps}

Let $\phi:(M, g) \rightarrow(N, \bar{g})$ be a map between Riemannian manifolds. We use $\phi^{-1}(T N)$ to denote the pull-back bundle of the tangent bundle $T N$ by $\phi$ (or the induced bundle of $\phi$ ). The pull-back bundle has base $M$ and the fiber $T_{\phi(p)} N$ over $p \in M$. The sections of $\phi^{-1}(T N)$ are called vector fields along $\phi$. The differential $\mathrm{d} \phi$ of $\phi$ can be regarded as a section of

$$
T^{*} M \otimes \phi^{-1}(T N)=\operatorname{Hom}\left(T M, \phi^{-1}(T N)\right) \rightarrow M .
$$

Let $\nabla^{\phi}$ denote the pull-back connection on $\phi^{-1}(T N)$ defined by

$$
\begin{gathered}
\nabla^{\phi}: \Gamma(T M) \times \Gamma\left(\phi^{-1}(T N)\right) \rightarrow \Gamma\left(\phi^{-1}(T N)\right), \\
\nabla_{X}^{\phi}\left(\phi^{*} \eta\right):=\nabla_{\mathrm{d} \phi(X)}^{N}(\eta)
\end{gathered}
$$

where $\phi^{*} \eta=\eta \circ \phi \in \Gamma\left(\phi^{-1}(T N)\right)$. Denote by $\Gamma\left(\phi^{-1}(T N)\right)$ the space of all sections of $\phi^{-1}(T N)$.

The second fundamental form $\sigma$ of a map $\phi:(M, g) \rightarrow(N, \bar{g})$ is given by the covariant differential $\nabla(d \phi)$. A map $\phi$ is called totally geodesic if its second fundamental form vanishes identically.

The energy density $e(\phi)$ of $\phi$ is a non-negative function given by

$$
e(\phi)_{u}=\frac{1}{2}\left\|d \phi_{u}\right\|^{2}=\frac{1}{2} \operatorname{tr}\left(\phi^{*} \bar{g}\right)_{u}, \quad u \in M,
$$

where $d \phi$ denotes the differential of $\phi,\|d \phi\|$ is the Hilbert-Schmidt norm of $d \phi$, and " $\operatorname{tr}^{\prime \prime}$ denotes the trace. The Dirichlet energy of $\phi$ over any compact domain $D$ of $M$ is defined by

$$
E(\phi, D)=\int_{D} e(\phi) d V
$$

A variation of a map $\phi$ is a smooth map $F: M \times(-\varepsilon, \varepsilon) \rightarrow N$ such that $F(p, 0)=\phi(p), p \in M$. A variation $\left\{\phi_{t}\right\}$ is said to be supported in a domain $D$ if $\phi_{t}=\phi$ on $M \backslash \stackrel{\circ}{D}$ for all $t \in I$, where $\stackrel{D}{D}$ denotes the interior of $D$. Note that $\left.\frac{\partial \phi_{t}}{\partial t}\right|_{t=0}=V(p)$ defines a vector field on the pull back bundle $\phi^{-1}(T N)$ and it is called the vector field of the variation $\left\{\phi_{t}\right\}$.

It was well-known that for a smooth variation $\left\{\phi_{t}\right\}$ of $\phi:(M, g) \rightarrow(N, \bar{g})$ supported in $D$, we have the first variation (cf. [22])

$$
\left.\frac{d}{d t} E\left(\phi_{t}, D\right)\right|_{t=0}=-\int_{D}\langle V, \tau(\phi)\rangle d v_{g}
$$

where $V=\left.\frac{\partial \phi_{t}}{\partial t}\right|_{t=0}$ is the variation vector field, and $\tau(\phi)$ is the tension field of $\phi$ given by

$$
\tau(\phi)=\operatorname{div}(d \phi)
$$

\subsection{Harmonic Map and Tension Field}

Definition 1. [22] A map $\phi:(M, g) \rightarrow(N, \bar{g})$ between two Riemannian manifolds is called a harmonic map if it is a critical point of the energy functional $E(\phi, D)$ defined by (32), where $D$ is any compact domain of $M$. 
It follows form Definition 1 that a harmonic map $\phi:(M, g) \rightarrow(N, \bar{g})$ is a solution of the corresponding Euler-Lagrange equation for the Dirichlet energy functional. Thus, a map $\phi$ is harmonic if and only if its tension field $\tau(\phi)$ vanishes identically [22].

For a given map $\phi:(M, g) \rightarrow(N, \bar{g})$, let $\left\{x^{i}\right\}$ and $\left\{y^{\alpha}\right\}$ be the local coordinates on $M$, and $N$, respectively. Then

$$
\phi(x)=\left(\phi^{1}(x), \cdots, \phi^{n}(x)\right)
$$

will be the local expression of the map $\phi$. Let $\left\{\partial_{i}\right\}$ and $\left\{\partial_{\alpha}\right\}$ be the natural frames of the local coordinates on $M$ and $N$ respectively. For simplicity, we put $\phi_{i}^{\alpha}=\frac{\partial \phi^{\alpha}}{\partial x^{i}}$ and $\phi_{i j}^{\alpha}=\frac{\partial^{2} \phi^{\alpha}}{\partial x^{i} \partial x^{j}}$. Then, in terms of local coordinates, we have

$$
\tau(\phi)=g^{i j}\left(\phi_{i j}^{\gamma}-\Gamma_{i j}^{k} \phi_{k}^{\gamma}+\phi_{i}^{\alpha} \phi_{j}^{\beta}\left(\bar{\Gamma}_{\alpha \beta}^{\gamma} \circ \phi\right)\right) \partial_{\gamma},
$$

where $\Gamma_{i j}^{k}$ and $\bar{\Gamma}_{\alpha \beta}^{\gamma}$ denote the Christoffel symbols of $(M, g)$ and $(N, \bar{g})$ with respect to the local coordinate systems $\left\{x^{i}\right\}$ and $\left\{y^{\alpha}\right\}$, respectively.

Remark 1. Definition 1 makes sense for harmonic maps between pseudo-Riemannian manifolds as well.

\subsection{Second Variation of Energy}

Assume that $\phi:(M, g) \rightarrow(N, \bar{g})$ is a harmonic map from a compact manifold $M$ into $(N, \bar{g})$. Let

$$
F: I_{\varepsilon} \times I_{\mathcal{\varepsilon}} \times M \rightarrow N, \quad F(s, t, p)=\phi_{s, t}(p), \text { and } \phi_{0,0}(p)=\phi(p),
$$

be a smooth two parameter variation of $\phi$.

Let $v(p)=\left.\frac{\partial F}{\partial s}\right|_{(0,0, p)} \in T_{\phi(p)} N, w(p)=\left.\frac{d F}{d t}\right|_{(0,0, p)} \in T_{\phi(p)} N$ be the variation vector fields. Then the Hessian, $H_{\phi}$, of the energy at $\phi$ is defined by

$$
H_{\phi}(v, w)=\left.\frac{\partial^{2}}{\partial s \partial t} E\left(\phi_{s, t}\right)\right|_{(s, t)=(0,0)} .
$$

Then we have

Theorem 1. [23] If $\phi:(M, g) \rightarrow(N, \bar{g})$ is a harmonic map from a compact manifold. Then the Hessian of the energy at $\phi$ is given by

$$
\begin{aligned}
H_{\phi}(v, w) & =\int_{M}\left\{\left\langle\nabla^{\phi_{v}}, \nabla^{\phi_{w}}\right\rangle-\operatorname{tr}_{g}\left\langle K^{N}(d \phi(\cdot), v) d \phi(\cdot), w\right\rangle\right\} d v_{g} \\
& =\int_{M}\left\langle J_{\phi} v, w\right\rangle d v_{g}
\end{aligned}
$$

for vector fields $v, w$ along $\phi$, where $K^{N}$ denotes the curvature tensor of $(N, \bar{g})$ and the Jacobi operator $J_{\phi}$ is given by

$$
J_{\phi} v=-\operatorname{tr}_{g}\left(\nabla^{2} v\right)-\operatorname{tr}_{g} K^{N}(d \phi, v) d \phi .
$$

\subsection{Stability, Index and Nullity}

A vector field $v$ along $\phi$ satisfying $J_{\phi} v=0$ is called a Jacobi field. The index of $\phi$, denoted by $\operatorname{Ind}(\phi)$, is the dimension of the largest subspace of $\Gamma\left(\phi^{-1}(T N)\right)$ on which $H_{\phi}$ is negative definite.

A harmonic map $\phi$ is called stable if then index ind $(\phi)=0$. Geometrically, a harmonic map $\phi$ is stable if its energy integral $E(\phi)$ is a local minimum of $E\left(\phi_{t}\right)$ for any variation $\left(\phi_{t}\right)$ with $\phi_{0}=\phi$. Otherwise, it is called unstable.

The nullity of $\phi$, denoted by $\operatorname{Null}(\phi)$ is the dimension of the kernel of $J_{\phi}$. It follows from the spectral properties of elliptic operators on compact manifolds that both $\operatorname{Ind}(\phi)$ and $N u l l(\phi)$ are finite. 
For a Riemannian manifold $(M, g)$, let $\mathbf{i}(M)$ denote the space of all infinitesimal isometries on $(M, g)$. Then the Killing nullity of a harmonic map $\phi:(M, g) \rightarrow(N, \bar{g})$ is defined by

$$
\operatorname{Null}_{k}(\phi)=\operatorname{dim}(\operatorname{Span}(\mathbf{i}(N), d \phi(\mathbf{i}(M)),
$$

where $d \phi(\mathbf{i}(M))$ is considered as variation vector fields along $\phi$. The reduced nullity of $\phi$, denoted by $\operatorname{Null}_{r}(\phi)$, is defined by (see [23])

$$
\operatorname{Null}_{r}(\phi)=\operatorname{Null}(\phi)-\operatorname{Null}_{k}(\phi)
$$

The following two results on stable harmonic maps are well-known.

Theorem 2. [24] For $n \geq 3$, there exists no non-constant stable harmonic map from any compact Riemannian manifold to $S^{n}$.

Theorem 3. [25] For $n \geq 3$, there exists no non-constant stable harmonic map from $S^{n}$ to any Riemannian manifold.

\section{Identity Maps, Harmonic Metrics and Harmonic Tensors}

\subsection{Relative Harmonic Metrics}

Let $\phi:(M, g) \rightarrow(N, h)$ be an immersion and $g^{\prime}=\phi^{*} h$ be the Riemannian metric on $M$ induced via $\phi$. Let $\varphi:\left(M, g^{\prime}\right) \rightarrow(N, h)$ be the associated isometric immersion, and $i d_{M}:(M, g) \rightarrow\left(M, g^{\prime}\right)$ be the identity map. Then the tension field of the composition $\varphi \circ i d_{M}$ of $\operatorname{maps} \varphi$ and $i d_{M}$ is given by

$$
\tau\left(\varphi \circ i d_{M}\right)=d \phi\left(\tau\left(i d_{M}\right)\right)+\operatorname{tr}_{g} B
$$

where " $\operatorname{tr}_{g}$ " is the trace with respect to $g$ and $B$ is the second fundamental form of the associated isometric immersion $\varphi:\left(M, g^{\prime}\right) \rightarrow(N, h)$. Thus, it follows from (40) that the composition $\varphi \circ i d_{M}$ is a harmonic map if and only if the identity map $i d_{M}:(M, g) \rightarrow\left(M, g^{\prime}\right)$ is harmonic and $\operatorname{tr}_{g} B=0$.

Partly motivated by this fact, the author and Nagano introduced in 1984 the following notion of (relative) harmonic metrics on a Riemannian manifold.

Definition 2. [2] A metric $g^{\prime}$ on a Riemannian manifold $(M, g)$ is called harmonic with respect to $g$ if the identity $\operatorname{map}_{\mathrm{id}}:(M, g) \rightarrow\left(M, g^{\prime}\right)$ is a harmonic map.

Let $g$ and $g^{\prime}$ be metrics on an $n$-manifold $M$. Denote by $\nabla, \Gamma_{i j}^{k}, \cdots$, etc, the Levi-Civita connection, Christoffel symbols, $\cdots$, etc of $(M, g)$; and by $\nabla^{\prime}, \Gamma_{i j}^{\prime k}, \cdots$, etc, the corresponding quantities of $\left(M, g^{\prime}\right)$, respectively.

From (35) we find the following.

Lemma 1. A metric $g^{\prime}$ on $M$ is harmonic with respect to a metric $g$ on $M$ if and only if

$$
g^{j i}\left(\Gamma_{j i}^{\prime k}-\Gamma_{j i}^{k}\right)=0 .
$$

For the metric tensor $g^{\prime}$, if we put

$$
f=\operatorname{tr}_{g} g^{\prime}=g^{i j} g_{i j}^{\prime},
$$

then we have

$$
\nabla_{j} f=2 g_{i}^{\prime k} L_{j k}^{i}
$$


Put

$$
L_{j i}^{k}=\Gamma_{j i}^{\prime k}-\Gamma_{j i}^{k}
$$

Then $L=\left(L_{j i}^{k}\right)$ is a tensor of type $(1,2)$ satisfying $L_{j i}^{k}=L_{i j}^{k}$. Moreover, it follows from the definition of Christoffel symbols that

$$
\nabla_{k} g_{j i}^{\prime}=g_{j a} L_{k i}^{a}+g_{i a}^{\prime} L_{k j}^{a} .
$$

Now, we may state the following useful result from [2] which provides an easy way to determine whether a metric $g^{\prime}$ is harmonic with respect to a given one $g$ on a manifold $M$.

Proposition 1. Let $g$ and $g^{\prime}$ be two Riemannian metrics on a manifold $M$. Then $g^{\prime}$ is harmonic with respect to $g$ if and only if we have

$$
\nabla_{j} f=2 \omega_{i}
$$

where $f=\operatorname{tr}_{g} g^{\prime}$ and $\omega_{i}=\nabla^{k} g_{k i}^{\prime}$.

\subsection{Space of Harmonic Tensors}

Let $\mathcal{S}$ denote the space of all symmetric covariant tensor fields of degree 2 on a Riemannian manifold $(M, g)$. If we regard a tensor $T \in \mathcal{S}$ as an energy-momentum tensor, the equation $\delta T=0$ is known in [26] as the conservation law of energy momentum.

Let $\mathcal{M}$ denote the space of all Riemannian metrics on $(M, g)$. We put

$$
\mathcal{H}_{g}=\left\{g^{\prime} \in \mathcal{M}: g^{\prime} \text { is harmonic with respect to } g\right\} .
$$

Then $\mathcal{H}_{g}$ is a star set centered at $g$ in the space $\mathcal{S}$ (see [2]). The following notion of (relative) harmonic tensors was introduced in [2].

Definition 3. Let $(M, g)$ be a Riemannian manifold. A tensor $T \in \mathcal{S}$ on $(M, g)$ is called a harmonic tensor with respect to $g$ if it satisfies (46).

The next two theorems describe the space of all harmonic tensors with respect to $g$.

Theorem 4. [2] Let $(M, g)$ be a Riemannian manifold of dimension $\geq 3$. Then we have the following linear isomorphism of vector spaces:

$$
\left\{g^{\prime} \in \mathcal{S}: g^{\prime} \text { is harmonic w.r.t. } g\right\} \cong \operatorname{ker}(\delta) \equiv\{T \in \mathcal{S}: \delta T=0\} .
$$

This isomorphism is given by $\mathcal{S} \ni g^{\prime} \mapsto g^{\prime}-\frac{1}{2}\left(\operatorname{tr}_{g} g^{\prime}\right) g \in \operatorname{ker}(\delta)$.

Theorem 5. [2] Let $(M, g)$ be a Riemannian 2-manifold. Then

$$
\left\{g^{\prime} \in \mathcal{S}: g^{\prime} \text { is harmonic w.r.t. } g\right\}=\{\lambda g: \lambda \in \mathcal{F}(M)\} \oplus\{T \in \operatorname{ker}(\delta): \operatorname{tr} T=0\},
$$

where $\mathcal{F}(M)$ is the space of all smooth functions on $M$.

Remark 2. Theorems 4 and 5 imply that the space of all harmonic tensors with respect to $g$ is infinite-dimensional.

The following theorem follows from Proposition 1 and the second Bianchi identity (see [2] page 399). 
Theorem 6. Let $(M, g)$ be Riemannian manifold. Then

(a) The Ricci tensor of $(M, g)$ is a harmonic tensor with respect to $g$.

(b) The Ricci tensor of $(M, g)$ is co-closed if and only if $(M, g)$ has a constant scalar curvature.

In particular, Theorem 6 implies the following.

Corollary 1. Let $(M, g)$ be a Riemannian manifold whose Ricci tensor Ric is definite. Then the identity map $i d_{M}:(M, g) \rightarrow(M$, Ric $)$ is harmonic.

For 2-dimensional manifolds, we also have the following.

Theorem 7. [2] If $(M, g)$ is an orientable Riemannian 2-manifold, then $\left\{T \in \operatorname{ker}(\delta): \operatorname{tr}_{g} T=0\right\}$ is linearly isomorphic with the space of holomorphic quadratic differentials on $M$ with the natural complex structure.

From Theorem 7 and Riemann-Roch's theorem, we have

Corollary 2. If $M$ is a 2-sphere or a real projective plane, then we have $\left\{g^{\prime} \in \mathcal{S}: g^{\prime}\right.$ is harmonic w.r.t. $\left.g\right\}=$ $\{\lambda g: \lambda \in \mathcal{F}(M)\}$.

Remark 3. One of the reviewers of this article pointed out that there is a discussion of the harmonicity of the identity map and its relation to DeTurck's method for breaking gauge invariance by Graham and Lee [27] published in 1991.

\subsection{Links between Geodesic Vector Fields and Harmonic Tensors}

A Killing vector field on an orientable compact Riemannian manifold is characterized by the following two conditions (see [28]):

(1) $\quad g^{j i} \nabla_{j} \nabla_{i} v^{k}+R i c_{i}^{k} v^{i}=0$ and

(2) divergence $\nabla_{i} v^{i}=0$.

On the other hand, the notion of geodesic vector fields was introduced by Yano and Nagano in [29] as follows.

Definition 4. A vector field $v=\left(v^{i}\right)$ on a Riemannian manifold $(M, g)$ is called a geodesic vector field if it satisfies ${ }^{j i} \nabla_{j} \nabla_{i} v^{k}+R i c_{i}^{k} v^{i}=0$.

The next theorem provides a simple link between geodesic vector fields and harmonic metrics.

Theorem 8. [2] A vector field $v$ on a Riemannian manifold $(M, g)$ is a geodesic vector field if and only if $g^{\prime}=\mathcal{L}_{v} g$ is a harmonic tensor with respect to $g$, where $\mathcal{L}_{v}$ denotes the Lie derivative with respect to $v$.

Remark 4. Analogous to Definition 2, a pseudo-Riemannian metric $g^{\prime}$ on a manifold $M$ is said to be harmonic

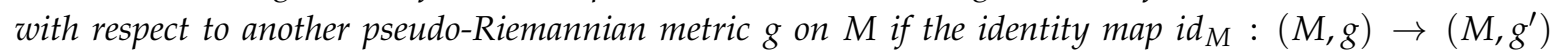
is harmonic.

It was shown in [30] that the results given in Sections 4.2 and 4.3 hold true in pseudo-Riemannian setting. Some further results on harmonic metrics in pseudo-Riemannian setting can also be found in [31,32] by Bejan and Duggal.

\subsection{A Volume-Decreasing Phenomenon}

If $g^{\prime}$ is a co-closed harmonic metric with respect to a given metric $g$ on a Riemannian manifold $M$, then we have 
(i) $\operatorname{tr}_{g} g^{\prime}$ is constant and

(ii) for any positive number $c, c g^{\prime}$ is also a co-closed harmonic metric with respect to $g$.

For co-closed harmonic metrics, we have the following volume-decreasing phenomenon.

Theorem 9. [2] Let $(M, g)$ is Riemannian manifold. If $g^{\prime}$ is a co-closed harmonic metric w.r.t. $g$ on $M$ satisfying $\operatorname{tr}_{g} g^{\prime}=\operatorname{tr}_{g} g$, then

(1) The volume form $d v_{g^{\prime}} \leq d v_{g}$ at each point of $M$; hence the identity map $i_{M}:(M, g) \rightarrow\left(M, g^{\prime}\right)$ is volume-decreasing.

(2) $d v_{g^{\prime}}=d v_{g}$ on $M$ if and only if $g^{\prime}=g$ on $M$.

\subsection{Identity Maps on Warped Products}

Let $\left(B, g_{1}\right)$ and $\left(N, g_{2}\right)$ be Riemannian manifolds and $f$ a positive function on $B$. Then the product manifold $B \times N$ equipped with the warped product metric $g=g_{1}+f^{2} g_{2}$ is called a warped product manifold with $f$ as its warping function. We denote this warped product manifold by $B \times{ }_{f} N$.

Pyo, Kim and Park proved the following result in [33].

Theorem 10. Let $\left(B, g_{1}\right)$ and $\left(N, g_{2}\right)$ be Einstein manifolds such that the Ricci tensors $R^{B}$ and $R^{N}$ of $B$ and $N$ satisfy $R^{B}=c_{1} g_{1}$ and $R^{N}=c_{2} g_{2}$, respectively. If the identity map

$$
i d_{B \times N}:\left(B \times N, g_{1}+g_{2}\right) \rightarrow\left(B \times N, g_{1}+f^{2} g_{2}\right)
$$

is harmonic, then $B \times{ }_{f} N$ is Einstein if and only if $c_{1}=f^{2} c_{2}$.

Remark 5. Eells and Sampson proved in [22] that the identity map id $d_{S^{m}}$ of an $m$-sphere $S^{m}(m \geq 3)$ is deformable to maps of arbitrary small energy. For further results on identity maps from homotopic points of view, we refer to the two reports [34,35] by Eells and Lemaire.

4.6. Identity Maps on Kaehler and Hyper-Kaehler Manifolds

Let $(M, J, g)$ be a Kaehler manifold. Then, with respect to a system of local coordinates $\left(x^{h}\right)$, we have

$$
g_{r s} J_{j}^{r} J_{i}^{S}=g_{j i}, \quad \nabla_{k} J_{j}^{i}=0 .
$$

The following proposition was proved in [36] by Watanabe and Dohira.

Proposition 2. Let $(M, J, g)$ be a Kaehler manifold. If a symmetric tensor $T$ on $(M, J, g)$ is represented by $T_{j i}=J_{j}^{k}\left(\nabla_{i} \eta_{k}-\nabla_{k} \eta_{i}\right)$ for some 1-form $\eta=\left(\eta_{i}\right)$, then $\mathrm{T}$ is a harmonic tensor with respect to $\mathrm{g}$.

Watanabe and Dohira also considered in [36] two Kaehler structures $(J, g)$ and $\left(J, g^{\prime}\right)$ on a complex manifold $M$. Let $\Omega$ and $\Omega^{\prime}$ denote the Kaehler forms corresponding to $g$ and $g^{\prime}$, respectively. By applying Proposition 2, they proved following.

Theorem 11. If $\Omega^{\prime}$ belongs to the Kaehler class $[\Omega]$, then the identity map id $d_{M}:(M, J, g) \rightarrow\left(M, J, g^{\prime}\right)$ is harmonic, i.e., $g^{\prime}$ is harmonic with respect to $g$.

Proposition 2 was extended to hyper-Kaehler manifolds by A. D. Vîlcu and G. E. Vîlcu as follows.

Theorem 12. [37] Let $(M, g, I, J, K)$ be a hyper-Kaehler manifold. If a symmetric tensor $T$ is represented by

$$
T_{j i}=\sum_{\Phi=I, J, K} \Phi_{j}^{k}\left(\nabla_{i} \varphi_{k}-\nabla_{k} \varphi_{i}\right) .
$$


for some 1-form $\varphi=\left(\varphi_{i}\right)$, then $T$ is a harmonic tensor with respect to $g$.

\section{Walker Metrics and Identity Maps}

\subsection{Walker Manifolds}

Definition 5. A Walker manifold $(W, g, \mathcal{D})$ is a pseudo-Riemannian manifold $\left(W^{n}, g\right)$ equipped with a lightlike distribution $\mathcal{D}$ (i.e., $g$ restricted to $\mathcal{D}$ is zero) which is parallel with respect to the Levi-Civita connection of $g$ (cf. $[38,39])$.

In the 4-dimensional case, where the distribution $\mathcal{D}$ has the maximum dimension (i.e., when $\operatorname{dim} \mathcal{D}=2)$, the metric $g$ is of neutral signature and there exists an adapted local coordinates $(x, y, z, t)$ such that the matrix $Q$ associated with the metric $g$ takes the form

$$
Q=\left(\begin{array}{ll}
0 & I \\
I & S
\end{array}\right)
$$

where 0 and $I$ denote $2 \times 2$ zero and identity matrices, respectively; and

$$
S=\left(\begin{array}{ll}
a & c \\
c & b
\end{array}\right)
$$

with $a, b, c$ being functions of $(x, y, z, t)$.

\subsection{Equivalence Classes of Walker 4-Manifolds}

In [40], Bejan and Druţă-Romaniuc introduced an equivalence relation on the set of all Walker metrics on a 4-dimensional manifold as follows: Two Walker metrics $g$ and $\hat{g}$ on a Walker 4-manifold $W^{4}$ are said to be equivalent, denoted by $g \sim \hat{g}$, if around any point there exist some adapted local coordinates $(x, y, z, t)$ with respect to which the entries of the associated matrices $Q_{a, b, c}$ of $g$ and $\hat{Q}_{\hat{a}, \hat{b}, \hat{c}}$ of $\hat{g}$ satisfy the following relation:

$$
\left\{\begin{array}{l}
\hat{a}=a+\int \lambda_{y} d x+\alpha(y, z, t) \\
\hat{b}=b+\int \lambda_{x} d y+\beta(x, z, t) \\
\hat{c}=c-\lambda(x, y, z, t)
\end{array}\right.
$$

for some local smooth functions $\alpha(y, z, t), \beta(x, z, t)$ and $\lambda(x, y, z, t)$.

Example 1. Let $\mathcal{M}$ denote the set of all Walker metrics on $\mathbb{R}^{4}$, whose associated matrices with respect to the coordinate frame are given by (47). Then we may identify $\mathcal{M}$ with $Q_{a, b, c}\left(a, b, c \in \mathcal{F}\left(\mathbb{R}^{4}\right)\right)$. Consider the group

$$
G=\mathcal{F}\left(\mathbb{R}^{3}\right) \times \mathcal{F}\left(\mathbb{R}^{3}\right) \times \mathcal{F}\left(\mathbb{R}^{4}\right)
$$

with respect to the additive operation. Then $G$ acts on $\mathcal{M}$ by the relation (49), and " $\sim$ " is the equivalence relation whose classes are the orbits.

On the canonical Walker space $\left(\mathbb{R}^{4}, g_{0}, \mathcal{D}\right)$ with a canonical neutral metric $g_{0}$, the set of all Walker metrics harmonic with respect to $g_{0}$ is in one-to-one correspondence with the transformation group G from (50) (see [40]).

\subsection{Relative Harmonic Metrics on Walker 4-Manifolds}

Bejan and Drctă-Romaniuc determined all Walker metrics which are harmonic with respect to a given Walker metric on a Walker 4-manifold. 
Theorem 13. [40] Let $\left(W^{4}, g, \mathcal{D}\right)$ be a Walker 4-manifold. Then, for any Walker metric $\hat{g}$ on $W^{4}$, the identity map id $_{W^{4}}:\left(W^{4}, g, \mathcal{D}\right) \rightarrow\left(W^{4}, \hat{g}, \mathcal{D}\right)$ is harmonic if and only if $g \sim \hat{g}$.

\section{Index and Nullity of Identity Maps}

In this section we present results on nullity and stability on identity maps.

\subsection{Nullity of Identity Maps}

Let us consider the identity map $i d_{M}:(M, g) \rightarrow(M, g)$. Lemma 1 shows that $i d_{M}$ is always harmonic. In this case, the second variation of the harmonic $i d_{M}:(M, g) \rightarrow(M, g)$ is given by

$$
H_{i d_{M}}(v, v)=\int_{M}\{\langle\nabla v, \nabla v\rangle-\operatorname{Ric}(v, v)\} d v
$$

for vector fields $v$ on $M$. Thus, the nullity of $i d_{M}:(M, g) \rightarrow(M, g)$ is the dimension of the space of the vector fields $v$ satisfying

$$
H_{i d_{M}}(u, v)=\int_{M}\{\langle\nabla u, \nabla v\rangle-\operatorname{Ric}(u, v)\} d v=0
$$

for any vector field $u$ with compact support in $M$. The following useful formula can be found in ([28] page 57).

$$
H_{i d_{M}}(v, v)=\int_{M}\left\{\frac{1}{2}\left|\mathcal{L}_{v} g\right|^{2}-(\operatorname{div} v)^{2}\right\} d v_{g}
$$

where $\operatorname{div} v$ denotes the divergence of $v$.

We have the following general result.

Theorem 14. [2] Let $(M, g)$ be a Riemannian manifold. Then the nullity of the identity map id $d_{M}:(M, g) \rightarrow$ $(M, g)$ is equal to the dimension of $\operatorname{ker}(\delta)^{\perp}=\left\{G \in \mathcal{S}: G=\mathcal{L}_{v} g\right.$ for some geodesic vector field $\left.v\right\}$.

Theorem 14 implies the following.

Corollary 3. If $(M, g)$ be a compact Riemannian manifold, then $\{G \in \mathcal{S}: G$ is harmonic w.r.t. $g$ and $G \perp$ $\operatorname{ker}(\delta)\}$ is finite-dimensional.

Corollary 4. Let $(M, g)$ be a compact irreducible symmetric space. Then the nullity of the identity map id $d_{M}:(M, g) \rightarrow(M, g)$ is greater than the dimension of the isometry group if and only if $(M, g)$ is either a Hermitian symmetric space or the exceptional simple group manifold $G_{2}$.

Corollary 4 follows from combining Theorem 14 and a result in [41] given by of Yano and Nagano.

\subsection{Index and Relative Nullity of Identity Maps}

In view of (53), Smith [23] obtained the following.

Proposition 3. Let $(M, g)$ be a compact oriented Riemannian n-manifold with $n \geq 3$. Then the index of $i d_{M}:(M, g) \rightarrow(M, g)$ satisfies

$$
\operatorname{index}\left(i d_{M}\right) \geq \operatorname{dim} \operatorname{ci}(M),
$$

where $\mathbf{c i}(M)$ denotes the space of infinitesimally conformal vector fields on a Riemannian manifold $(M, g)$.

Let $\Delta_{H}$ be the Hodge Laplacian acting on vector fields via duality. Then the Jacobi operator $J_{i d_{M}}$ satisfies

$$
J_{i d_{M}} v=\Delta_{H} v-\operatorname{Ric}(v)
$$


where the Ricci tensor Ric of $M$ is regarded as a linear map on the tangent bundle TM. In particular, if $(M, g)$ is Einstein, (54) becomes

$$
J_{i d_{M}}=\Delta_{H}-2 c I,
$$

where $I$ denotes the identity operator and $c$ is a constant. By applying this formula, Smith [23] was able to relate the index and nullity of the identity map $i d_{M}$ to the eigenvalues of the Laplacian $\Delta$ acting on the space of smooth functions $\mathcal{F}(M)$.

By putting $\lambda(r)=\#\{$ eigenvalues $\lambda: 0<\lambda<r\}$ and $m(r)$ the multiplicity of $r$ with $m(0)=0$, Smith obtained the following result in [23].

Theorem 15. Let $(M, g)$ be a compact oriented Einstein manifold with Ricci tensor Ric $=c g$ for some constant $c$. Then the index, nullity and the relative nullity of the identity map id $d_{M}:(M, g) \rightarrow(M, g)$ satisfy

(1) $\operatorname{index}\left(i d_{M}\right)=\lambda(2 c)$,

(2) $\operatorname{Null}\left(i d_{M}\right)=\operatorname{dim} \mathbf{i}(M)+m(2 c)$,

(3) $\mathrm{Null}_{r}\left(i d_{M}\right)=m(2 c)$,

In particular, Theorem 15 implies the following.

Corollary 5. Let $(M, g)$ be a compact Einstein manifold with Ricci tensor Ric $=c g$ for some constant $c$. Then, the identity map id ${ }_{M}$ of $(M, g)$ is stable if and only if the first positive eigenvalue $\lambda_{1}$ of the Laplacian $\Delta$ acting on $\mathcal{F}(M)$ satisfies $\lambda_{1} \geq 2 c$.

\subsection{Index and Nullity of Spheres and Flat Torus}

Smith determined in [23] the index, relative nullity and Killing nullity of the identity maps of $S^{n}$ :

$$
\begin{aligned}
& \operatorname{index}\left(i d_{S^{n}}\right)= \begin{cases}0 & \text { if } n=1,2 \\
n+1 & \text { if } n=3\end{cases} \\
& \operatorname{Null}_{r}\left(i d_{S^{n}}\right)= \begin{cases}0 & \text { if } n \neq 2 \\
n+1 & \text { if } n=2 ;\end{cases} \\
& \operatorname{Null}_{k}\left(i d_{S^{n}}\right)=\frac{n(n+1)}{n}(n \geq 1)
\end{aligned}
$$

and for the flat torus $T^{n}$, he obtained

$$
\begin{array}{ll}
\text { index }_{\left(i d_{T^{n}}\right.}=0 & \text { for all } n \geq 2, \\
\operatorname{Null}_{r}\left(i d_{T^{n}}\right)=0 & \text { for all } n \geq 2, \\
\operatorname{Null}_{k}\left(i d_{T^{n}}\right)=n & \text { for all } n \geq 2 .
\end{array}
$$

\subsection{Index and Nullity of Product Maps}

Let $\phi_{i}:\left(M_{i}, g_{i}\right) \rightarrow\left(N_{i}, \bar{g}_{i}\right) i=1,2$, be two harmonic maps. Then the product map

$$
\phi:\left(M_{1} \times M_{2}, g_{1} \times g_{2}\right) \rightarrow\left(N_{1} \times N_{2}, \bar{g}_{1} \times \bar{g}_{2}\right)
$$

of $\phi_{1}, \phi_{2}$ is the map which carries $(x, y)$ in $M_{1} \times M_{2}$ to $\left(\phi_{1}(x), \phi_{2}(y)\right)$ in $N_{1} \times N_{2}$. The product map of two harmonic maps is also harmonic.

Fardoun and Ratto studied the index of nullity of product maps and obtained the following.

Proposition 4. [42] Let $(M, g)$ be a compact oriented Einstein manifold with Ric $=c g$ for some constant $c$. Then the index, nullity and the relative nullity of the identity map $i_{M}:(M, g) \rightarrow(M, g)$ satisfy

(1) $\operatorname{index}(\phi)=\operatorname{index}\left(\phi_{1}\right)+\operatorname{index}\left(\phi_{2}\right)$, 
(2) $\operatorname{Null}(\phi)=\operatorname{Null}\left(\phi_{1}\right)+\operatorname{Null}\left(\phi_{2}\right)$.

Additionally, in [42], Fardoun and Ratto determined the index and nullity of the identity maps for $S^{n} \times S^{m}$ and $S^{n} \times T^{m}$.

For $S^{n} \times T^{m}$, they obtained the following.

Theorem 16. [42] For the identity map id $d_{S^{n} \times T^{m}}$ of the product manifolds $S^{n} \times T^{m}$, we have:

$$
\begin{aligned}
& \text { (a) index }\left(i d_{S^{2} \times T^{m}}\right)=0, \quad m \geq 2 ; \\
& \text { (b) index }\left(i d_{S^{n} \times T^{m}}\right)=(n+1) \sum_{k=1}^{N} \#\left\{z \in \mathbb{Z}^{m}:|z|^{2}=k^{2}\right\}, m \geq 2, n>2 ; \\
& \text { (c) } \operatorname{Null}_{k}\left(i d_{S^{n} \times T^{m}}\right)=m+\frac{1}{2} n(n+1), m, n \geq 2 ; \\
& \text { (d) } \operatorname{Null}_{r}\left(i d_{S^{n} \times T^{m}}\right)= \begin{cases}3 & \text { if } n=2, m \geq 2, \\
0 & \text { if } n>2, m \geq 2,\end{cases}
\end{aligned}
$$

where $N=\max \left\{k \in N-\{0\}: 4 \pi^{2} k^{2}<n-2\right\}$.

\section{Identity Maps on Compact Symmetric Spaces}

If the identity map $i d_{M}:(M, g) \rightarrow(M, g)$ is stable, we simply say that the Riemannian manifold $M$ is stable. Otherwise, $M$ is said to be unstable. It is well-known that $M$ is stable whenever $M$ is Kaehlerian [23] (see also [1] page 131). Theorems 2 and 3 imply that the standard $n$-sphere $S^{n}$ is unstable for $n \geq 3$.

\subsection{Stability of Compact Symmetric Spaces}

The following result was obtained by combining Theorem 14 and Nagano's table of spectra given in [43].

Theorem 17. [23] The classical compact irreducible symmetric spaces are stable with the following exceptions: (1) $S^{n}, n \geq 3,(2) S p(p+q) / S p(p) \times S p(q)$ and (3) $S U(2 m) / S p(m), m>1$.

For exceptional symmetric spaces, Nagano proved in [1] that the Cayley projective plane $F_{4} / \operatorname{Spin}(9)$ is also unstable.

In [44], Ohnita proved the following two theorems.

Theorem 18. Let $M$ be an n-dimensional compact irreducible symmetric space. Denote by $\lambda_{1}$ and $c$ the first eigenvalue of the Laplacian on functions and the scalar curvature of $M$ respectively. Then the identity map $i d_{M}$ is unstable if and only if one of the following three conditions holds:

(a) $\lambda_{1}<2 c / n$.

(b) There exists no non-constant stable harmonic map from $M$ to any Riemannian manifold.

(c) There exists no non-constant stable harmonic map from any compact Riemannian manifold to $M$.

Theorem 19. Let $M$ be a simply connected compact irreducible symmetric space. Then the identity map id ${ }_{M}$ is unstable if and only if $M$ belongs to the following list:

(i) Simple Lie groups of type $A_{n}(n \geq 2), B_{2}$ and $C_{n}(n \geq 3)$;

(ii) $S U(2 n) / S p(n)(n \geq 3)$;

(iii) $S^{n}(n \geq 3)$;

(iv) $S p(p+q) / S p(p) \times S p(q)(p \geq q \geq 1)$;

(v) $E_{6} / F_{4}$;

(vi) $F_{4} / \operatorname{Spin}(9)$. 
Remark 6. Theorem 18 shows that a compact irreducible symmetric space is simply-connected if its identity map is unstable.

For stable compact symmetric space, Nagano [1] proved the following.

Theorem 20. A compact symmetric space $M$ is stable if and only if the Casimir operator (in the sense of [45]) acting on the tangent bundle TM or on the functions has no eigenvalue $<-1$.

Theorem 21. A compact simple, simply-connected Lie group $M$ is stable if and only if it is the exceptional group $E_{8}$.

Theorem 22. A compact connected simple Lie group $M$ with trivial center is stable if and only if it is the adjoint group of $S U(n), S O(2 n), E_{6}, E_{7}$ or $E_{8}$.

\subsection{Instability of Compact Symmetric Spaces}

Nagano proved the following properties for unstable symmetric spaces.

Theorem 23. Let $M=G / K$ be a unstable simply-connected symmetric space with compact and simple Lie group G. Then $M$ has the following properties:

(1) $M$ admits a totally geodesic immersion $\varphi$ into another symmetric space $M^{\prime}=G^{\prime} / K^{\prime}$ such that (i) $K^{\prime}=G$ or $S O(2) \times G$ and (ii) $\varphi(M)$ is a connected component of the fixed point set $M_{+}^{\prime}$ of the symmetry $s_{x}$ at some point $x$ of $M^{\prime}$ or this is true if one projects $\varphi(M)$ into a symmetric space of which $M^{\prime}$ is a covering space;

(2) The non-compact form $G^{\prime \prime}$ of $G^{\prime}$ with respect to $G^{\prime} / M^{\prime}$ can act on $M$ and as such contains $G$ properly;

(3) The submanifold $\varphi(M)$ is unstable as a minimal submanifold of $M^{\prime}$.

Remark 7. For geometric theory of the polars $\left(M_{+}\right)$as well as of the meridians $\left(M_{-}\right)$of compact symmetric spaces, see [46-48].

\section{Identity Maps of Other Spaces}

8.1. Identity Maps on Homogeneous Space $(S U(3) / T(k, \ell), g)$

Let $i=\sqrt{-1}$ and put

$$
T(k, \ell)=\left\{\operatorname{diag}\left[e^{2 \pi i k \theta}, e^{2 \pi i \ell \theta}, e^{-2 \pi i(k+\ell) \theta}\right]: \theta \in \mathbf{R},|k|+|\ell| \neq 0(k, \ell \in \mathbb{Z})\right\} .
$$

Then $\operatorname{SU}(3) / T(k, \ell)$ is a non-symmetric 7-dimensional homogeneous space which admit a positively curved Riemannian metric (cf. [49]).

Park [50] proved the following result for $S U(3) / T(k, \ell)$.

Theorem 24. Let $S U(3) / T(k, \ell)$ have the SU(3)-invariant metric $g$ which is canonically induced from an $A d(S U(3))$-invariant inner product on the Lie algebra $s u(3)$. If $k$ and $\ell$ are relatively prime, then the identity map of $(S U(3) / T(k, \ell), g)$ is stable.

\subsection{Stability of Spheres with Deformed Metrics}

From Section 6, we know that the standard unit $m$-sphere $\left(S^{m}, g_{0}\right)$ is unstable for $m \geq 3$. In [51], Tanno studied the stability of a family of deformed metrics $g(t)$ on $S^{m}$ with $m=2 n+1$ as follows:

For $m=2 n+1$, we have the Hopf fibration $\pi:\left(S^{m}, g_{0}\right) \rightarrow\left(C P^{n}, h_{0}\right)$, where $\left(C P^{n}, h_{0}\right)$ is the complex projective $n$-space of constant holomorphic sectional curvature 4 . Let $\xi$ be a vector field on $S^{m}$ which is tangent to the fibers and of unit length. Then $\xi$ is a Killing vector field with respect to the standard metric $g_{0}$ and let $\eta$ be 1 -form dual to $\xi$ with respect to $g_{0}$. 
Consider the one-parameter family of metrics $g(t)$ on $S^{m}$ is defined by

$$
g(t)=t^{-1} g_{0}+t^{-1}\left(t^{m}-1\right) \eta \otimes \eta,
$$

where $0<t<\infty$. It is known that the metric given by (56) on $S^{2 n+1}$ are homogeneous Riemannian metrics which are not symmetric nor Einstein. With respect to these metrics $(g(t))$, the volume elements remain unchanged (cf. [51,52]).

Tanno proved the following.

Theorem 25. [51] For $m=2 n+1 \geq 3$ and $t \in\left(t_{0}(m), \infty\right)$, the $m$-sphere $\left(S^{m}, g(t)\right)$ is unstable, where

$$
t_{0}(m)^{m}=\left[\left(m^{2}-4\right)^{2}-1\right] /\left(m^{2}-5\right) \text { and } t_{0}(3)=0.67 \cdots<t_{0}(m)<1 .
$$

Analogously, for $m=4 r+3$, S. Tanno [53] applied the Hopf fibration

$$
\pi:\left(S^{4 r+3}, g_{0}\right) \rightarrow\left(H P^{n}, h_{0}\right)
$$

with the corresponding Sasakian three-structure $\left\{\eta_{1}, \eta_{2}, \eta_{3}\right\}$ to define a one-parameter family of metric $g(t)$ on $S^{4 r+3}$ by

$$
g(t)=t^{-1} g_{0}+t^{-1}\left(t^{m / 3}-1\right) \sum_{\alpha} \eta_{\alpha} \otimes \eta_{\alpha}
$$

Then he proved the following instability result.

Theorem 26. [51] We have:

(a) For $m=4 r+3=7,11$, the sphere $\left(S^{4 r+3}, g(t)\right)$ is unstable.

(b) For $m=4 r+3 \geq 15$ and for $t \in\left(0, t_{0}(m)\right)$ or $t \in\left(t_{1}(m), \infty\right)$, the sphere $\left(S^{4 r+3}, g(t)\right)$ is unstable, where $t_{0}(m)$ and $t_{1}(m)$ are defined in $\S 4$ of [53].

\subsection{Identity Maps and Riemannian Submersions}

In Theorem 3, Xin gave an important result which says that each non-constant harmonic map from the unit $n$-sphere $S^{n}, n \geq 3$, into a Riemannian manifold is always unstable. Thus, Urakawa asked in [54] the following

Question 1. Does there exist a deformation $g_{t}, 0<t<\infty$, of the standard metric $g_{1}$ on $S^{n}$ such that if $g_{t}$ is far from $g_{1}$, then $\left(S^{n}, g_{t}\right)$ admits a stable harmonic map?

To provide an answer to this question, Urakawa [54] investigated a Riemannian submersion $\phi:(M, g) \rightarrow(N, h)$ with totally geodesic fibers. Then he considered the canonical variation $g_{t}, 0<$ $t<\infty$, of the metric $g$ which also gives a Riemannian submersion $\phi:\left(M, g_{t}\right) \rightarrow(N, h)$ with totally geodesic fibers. More precisely, Urakawa [54] proved the following theorem which gave an answer to this question.

Theorem 27. Assume that the identity map id ${ }_{N}$ of $(N, h)$ is stable. Then there exists a small number $\varepsilon$ such that, for every $0<t<\varepsilon$, the Riemannian submersion $\phi:\left(M, g_{t}\right) \rightarrow(N, h)$ is stable.

Since the identity map $i d_{C P^{n}}$ of the complex projective space $\left(C P^{n}, h\right)$ is stable, the Hopf fibering $\pi:\left(S^{2 n+1}, g_{t}\right) \rightarrow\left(C P^{n}, h\right)$ is stable for $0<t<\varepsilon$, for the canonical variation $g_{t}, 0<t<\varepsilon$, with $g_{1}=$ canonical metric. This provides an example which contrasts with the instability theorem of Xin (Theorem 3). Some further results on identity maps were also obtained by Urakawa in [54]. 


\subsection{Identity Maps on Generalized Sasakian Space Forms}

A Riemannian $(2 n+1)$-manifold $\left(\bar{M}^{2 n+1}, g\right)$ is called an almost contact metric manifold [55] if there exist a $(1,1)$ tensor field $\varphi$, a vector field $\xi$ (called the structure vector field), and a 1-form $\eta$ on $\bar{M}$ such that

$$
\eta(\xi)=1, \quad \phi^{2}(X)=-X+\eta(X) \xi, \varphi \xi=0, \quad \eta \circ \varphi=0,
$$

and

$$
g(\varphi X, \phi Y)=g(X, Y)-\eta(X) \eta(Y), \quad \eta(X)=g(X, \xi)
$$

for any vector fields $X, Y$ on $\bar{M}$.

An almost contact metric manifold $\left(\bar{M}^{2 n+1}, \varphi, \xi, \eta, g\right)$ is called a Sasakian manifold if it satisfies

$$
\left(\nabla_{X} \varphi\right) Y=g(X, Y) \xi-\eta(Y) X
$$

for $X, Y$ tangent to $\bar{M}^{2 n+1}$, where $\nabla$ is the Levi-Civita connection of $\bar{M}^{2 n+1}$. A Sasakian manifold with constant $\varphi$-sectional curvature $c$ is called Sasakian space form. In this case, the curvature tensor of $\bar{M}^{2 n+1}$ is given by

$$
\begin{aligned}
R(X, Y) Z= & \frac{c+3}{4}\{g(Y, Z) X-g(X, Z) Y\} \\
& +\frac{c-1}{4}\{g(X, \varphi Z) \varphi Y-g(Y, \varphi Z) \varphi X+2 g(X, \varphi Y) \varphi Z\} \\
& +\frac{c-1}{4}\{\eta(X) \eta(Z) Y-\eta(Y) \eta(Z) X \\
& +g(X, Z) \eta(Y) \xi-g(Y, Z) \eta(X) \xi\}
\end{aligned}
$$

for vector fields $X, Y, Z$ tangent to $\bar{M}^{2 n+1}$. Sasakian space forms can be modeled based on $c>-3$, $c=-3$ or $c<-3$.

Alegre, Blair and Carriazo introduced and studied in [56] the notion of a generalized Sasakian space form. An odd-dimensional manifold $\bar{M}^{2 n+1}$ equipped with an almost contact metric structure $(\phi, \xi, \eta, g)$ is called generalized Sasakian space form if there exist three functions $f_{1}, f_{2}, f_{3}$ on $\bar{M}^{2 n+1}$ such that

$$
\begin{aligned}
\bar{R}(X, Y) Z= & f_{1}\{g(Y, Z) X-g(X, Z) Y\} \\
& +f_{2}\{g(X, \varphi Z) \varphi Y-g(Y, \varphi Z) \varphi X+2 g(X, \varphi Y) \varphi Z\} \\
& +f_{3}\{\eta(X) \eta(Z) Y-\eta(Y) \eta(Z) X \\
& +g(X, Z) \eta(Y) \xi-g(Y, Z) \eta(X) \xi\} .
\end{aligned}
$$

We denote such a manifold by $\bar{M}^{2 n+1}\left(f_{1}, f_{2}, f_{3}\right)$. Obviously, a generalized Sasakian space form $\bar{M}^{2 n+1}\left(f_{1}, f_{2}, f_{3}\right)$ is a Sasakian space form if $f_{1}=\frac{c+3}{4}$ and $f_{2}=f_{3}=\frac{c-1}{4}$.

Rehman investigated in [57] the stability of generalized Sasakian space forms and obtained the following results.

Theorem 28. Let $\bar{M}^{2 n+1}\left(f_{1}, f_{2}, f_{3}\right)$ be a compact generalized Sasakian space form. If $\left(3 f_{2}+(2 n-1) f_{3}\right) \geq 0$ and $\left(3 f_{2}+2 n f_{1}-f_{3}\right) \leq 0$, then the identity map id $\bar{M}$ of $\bar{M}^{2 n+1}\left(f_{1}, f_{2}, f_{3}\right)$ is stable.

Theorem 29. Let $\bar{M}^{2 n+1}\left(f_{1}, f_{2}, f_{3}\right)$ be a compact domain of a generalized Sasakian space form such that $\left(3 f_{2}+(2 n-1) f_{3}\right) \geq 0$. If the first eigenvalue $\lambda_{1}$ of the Laplacian $\Delta$ acting on $\mathcal{F}(M)$ satisfies $\lambda_{1}<$ $2\left(3 f_{2}+2 n f_{1}-f_{3}\right)$, then the identity map id $\bar{M}$ of $\bar{M}^{2 n+1}\left(f_{1}, f_{2}, f_{3}\right)$ is unstable.

Theorems 28 and 29 imply the following corollaries, respectively. 
Corollary 6. Let $D$ be a compact domain of a Kenmotsu space form $M^{2 n+1}$ of constant $\varphi$-sectional curvature $c$. If $c$ lies in the open interval $\left(-1, \frac{3 n-1}{n+1}\right)$, then the identity map id $D$ of $D$ is stable.

Corollary 7. Let $M^{2 n+1}$ be a compact Sasakian space form of constant $\varphi$-sectional curvature $c \leq 1$. If the first eigenvalue of the Laplacian satisfies $\lambda_{1}<(n+1) c+3 n-1$, then the identity map id ${ }_{M}$ is unstable.

\section{Biharmonic Identity Maps}

\subsection{Biharmonic Maps and Biharmonic Submanifolds}

A biharmonic map is a map $\phi:(M, g) \rightarrow(N, h)$ between Riemannian manifolds that is a critical point of the bienergy functional

$$
E_{2}(\phi, g)=\frac{1}{2} \int_{D}|\tau(\phi)|^{2} d v_{g},
$$

where $D$ is any compact domain $D$ of $M$ and $\tau(\phi)$ is the tension field of the map $\phi$. The Euler-Lagrange equation of the bienergy functional was computed by Jiang in [58]. The first variational formula for the bienergy functional is given by

$$
\left.\frac{d E_{2}\left(\phi_{t}\right)}{d t}\right|_{t=0}=\int_{D}\left\langle\tau_{2}(\phi), \eta\right\rangle d V_{g}
$$

where $\eta$ is the variational vector field along $\phi$ and the bitension field $\tau_{2}(\phi)$ is given by

$$
\tau_{2}(\phi)=-\Delta^{\phi} \tau(\phi)-\operatorname{tr}_{g} K^{N}(d \phi(\cdot), \tau(\phi)) d \phi(\cdot),
$$

with $\Delta^{\phi} \tau(\phi)=\operatorname{tr}_{g}\left(\nabla_{\nabla^{M}}^{\phi}-\nabla^{\phi} \nabla^{\phi}\right) \tau(\phi)$ and $K^{N}$ being the curvature tensor of $(N, h)$. Hence, $\phi$ is biharmonic if and only if its bitension field $\tau_{2}(\phi)$ vanishes identically. A biharmonic submanifold of a Riemannian manifold is one whose defining isometric immersion is a biharmonic map. On the other hand, in his program to study finite type submanifolds, the author defined in the 1980s the notion of biharmonic submanifolds $M$ of Euclidean spaces as those whose position vector field $x$ satisfies $\Delta^{2} x=0$ (cf. $\left.[16,59,60]\right)$.

In 2002, Caddeo, Montaldo and Oniciuc shown in [61] that the condition $\tau_{2}(\phi)=0$ is equivalent to $\Delta^{2} x=0$ for Euclidean submanifolds. Thus, both definitions of biharmonicity coincide for Euclidean submanifolds.

\subsection{Biharmonic Maps and Relative Biharmonic Metrics}

The following definition was made in [62] (see also [63] page 449).

Definition 6. A Riemannian metric $\bar{g}$ on $M$ is called a (relative) biharmonic metric with respect to another metric $g$ if the identity map id $d_{M}:(M, g) \rightarrow(M, \bar{g})$ is biharmonic. A biharmonic metric $\bar{g}$ of $(M, g)$ is called proper biharmonic metric if it is non-harmonic.

Example 2. For $\lambda=|x|^{-1},|x|^{-2}, \frac{2}{1 \pm|x|^{2}}$, the conformally flat metrics $\lambda^{2} g_{0}$ is biharmonic with respect to Euclidean metric $g_{0}$ on an open set of $U \subset \mathbb{E}^{4}$, i.e., each of the following identity maps

$$
\begin{aligned}
& i d_{\mathbb{E}^{4}}:\left(\mathbb{E}^{4} \backslash\{0\}, g_{0}\right) \rightarrow\left(\mathbb{E}^{4} \backslash\{0\},|x|^{-2} g_{0}\right), \\
& i d_{\mathbb{E}^{4}}:\left(\mathbb{E}^{4} \backslash\{0\}, g_{0}\right) \rightarrow\left(\mathbb{E}^{4} \backslash\{0\},|x|^{-4} g_{0}\right), \\
& i d_{\mathbb{E}^{4}}:\left(\mathbb{E}^{4}, g_{0}\right) \rightarrow\left(\mathbb{E}^{4}, 4\left(1+|x|^{2}\right)^{-2} g_{0}\right), \\
& i d_{\mathbb{E}^{4}}:\left(\mathbb{B}^{4}, g_{0}\right) \rightarrow\left(\mathbb{B}^{4}, 4\left(1-|x|^{2}\right)^{-2} g_{0}\right),
\end{aligned}
$$


is a proper biharmonic map.

The purpose of this section is to present known results on biharmonicity of identity maps arisen from conformal change of metrics.

Theorem 30. [31] Let $M$ be an m-manifold. Then the identity map id $M:(M, g) \rightarrow\left(M, \bar{g}=e^{2 \rho} g\right)$ is biharmonic if and only if

$$
\begin{gathered}
0=\operatorname{tr}_{g} \nabla^{2}(\nabla \rho)+\left(-2 \Delta \rho+(2-m)|\nabla \rho|^{2}\right) \nabla \rho \\
+\frac{6-m}{2} \nabla\left(|\nabla \rho|^{2}\right)+\operatorname{Ric}^{g}(\nabla \rho) .
\end{gathered}
$$

Proposition 5. (cf. [63, Corollary 12.17]) Let M be an m-manifold. Then

(a) The identity map

$$
i d_{M}:(M, g) \rightarrow\left(M, \bar{g}=\lambda^{2} g\right),
$$

is a biharmonic map if and only if

$$
\lambda \nabla \Delta \lambda-3(\Delta \lambda) \nabla \lambda-\frac{m-4}{2} \nabla|\nabla \lambda|^{2}+2 \lambda \operatorname{Ric}^{g}(\nabla \lambda)=0,
$$

where Ric ${ }^{8}$ is the Ricci operator of $(M, g)$. In particular, for $m=4$, the identity map (64) is biharmonic if and only if

$$
\nabla(\lambda \Delta \lambda)-4(\Delta \lambda) \nabla \lambda+2 \lambda \operatorname{Ric}^{g}(\nabla \lambda)=0
$$

(b) The identity map

$$
i d_{M}:\left(M, \bar{g}=\lambda^{2} g\right) \rightarrow(M, g)
$$

is a biharmonic map if and only if

$$
\begin{gathered}
\lambda \nabla \Delta \lambda-3(\Delta \lambda) \nabla \lambda-3(m-6) \lambda^{-1}|\nabla \lambda|^{2} \nabla \lambda \\
+\frac{m-8}{2} \nabla|\nabla \lambda|^{2}+2 \lambda \operatorname{Ric}^{g}(\nabla \lambda)=0 .
\end{gathered}
$$

In particular, for $m=6$, the identity map (67) is biharmonic if and only if

$$
\lambda \nabla \Delta \lambda-3(\Delta \lambda) \nabla \lambda-\nabla|\nabla \lambda|^{2}+2 \lambda \operatorname{Ric}^{g}(\nabla \lambda)=0 .
$$

From Definition 6 and Proposition 5, we have

Corollary 8. A conformally related metric $\bar{g}=\lambda^{2} g$ on a Riemannian manifold $\left(M^{m}, g\right)$ with $m \geq 3$ is biharmonic with respect to $g$ if and only if the function $\lambda$ is solution of (65). In particular, a conformally related metric $\bar{g}=\lambda^{2} g$ on a 4-manifold $\left(M^{4}, g\right)$ is biharmonic with respect to $g$ if and only if the function $\lambda$ solves (66).

A conformally related metric $\bar{g}=e^{2 \gamma} g$ is called biharmonic in [62] if the identity map $i d_{M}:(M, \bar{g}) \rightarrow(M, g)$ is biharmonic. Thus, by statement (b) of Proposition 5, the equation for the "biharmonic metric" defined in [62] is Equation (68).

Corollary 9. [64] A conformally flat metric $h=\lambda^{2} g_{0}$ on an open set $U \subseteq \mathbb{E}^{4}$ is biharmonic if and only if the conformal factor is a solution of the Yamabe equation or, equivalently, the Riemannian manifold $\left(U, h=\lambda^{2} g_{0}\right)$, has constant scalar curvature $\rho_{h}$ and $\Delta \lambda=-\frac{\rho_{h}}{6} \lambda^{3}$. 
The 4-dimensional conformally flat space $\left(\mathbb{E}^{4} \backslash\{0\},|x|^{-2} g_{0}\right)$ appeared in a study of Yang-Mills equation in [65]. The following corollary shows that this conformally flat metric is one of the proper biharmonic metrics in a Euclidean 4-space.

Corollary 10. The conformally flat metric $h=|x|^{2 \alpha} g_{0}$ on $\mathbb{E}^{4} \backslash\{0\}$ is proper biharmonic with respect to Euclidean metric $g_{0}$ if and only if $\alpha=-1$, or -2 .

\subsection{Obstructions to Biharmonic Metrics on Einstein Manifolds}

The next three results of Baird, Fardoun and Ouakkas provide obstructions to the existence of proper biharmonic metric on compact Einstein manifolds.

Theorem 31. [66] Let $\left(M^{m}, g\right)$ be a compact Einstein manifold $\left(M^{m}, g\right)$ with Ric $=c g$. If $m \geq 6$ and $c<0$, or $m>6$ and $c \leq 0$, then the identity map id $M_{M^{m}}:\left(M^{m}, g\right) \rightarrow\left(M^{m}, e^{2 \rho} g\right)$ is biharmonic if and only if it is harmonic, i.e., $\rho$ is constant.

Theorem 32. [66] Let $\left(M^{m}, g\right)$ be a compact Einstein manifold with $m>2$ and Ric $=c g$. Assume that $c \geq 0$ and the 1 -form $\alpha=d \rho$ satisfies

$$
\frac{10-m}{2} \int_{M}|\alpha|^{2} \delta \alpha d v_{g}+(2-m) \int_{M}|\alpha|^{4} d v_{g}+2 c \int_{M}|\alpha|^{2} d v_{g} \leq 0 .
$$

Then id $M_{M^{m}}:\left(M^{m}, g\right) \rightarrow\left(M^{m}, e^{2 \rho} g\right)$ is biharmonic if and only if $i d_{M^{m}}$ is harmonic.

Remark 8. Given an arbitrary 1-form $\alpha$, one can always find a constant $k$ such that $k \alpha$ satisfies condition (70).

Theorem 33. [66] Let $(M, g)$ be a compact Einstein 10-manifold $(M, g)$ with Ric $=c g, c>0$. Then

(1) If $c \int_{M}|\alpha|^{2} d v_{g} \leq 4 \int_{M}|\alpha|^{4} d v_{g}$, then id $d_{M^{m}}:\left(M^{m}, g\right) \rightarrow\left(M^{m}, e^{2 \rho} g\right)$ is biharmonic if and only if it is harmonic.

(2) If $\frac{c}{4}(\operatorname{vol}(M))^{1 / 2} \leq\left(\int_{M}|\alpha|^{4} d v_{g}\right)^{1 / 2}$, then $i d_{M^{m}}:(M, g) \rightarrow\left(M, e^{2 \rho} g\right)$ cannot be biharmonic.

\subsection{A Biharmonicity Reduction Theorem For Submersions}

The next result was obtained by Balmuş.

Theorem 34. [31] Let $\pi:(M, g) \rightarrow(N, h)$ be a harmonic Riemannian submersion and $\tilde{h}$ a Riemannian metric on $N$. Denote by $\tilde{\pi}=i d_{N} \circ \pi:(M, g) \rightarrow(N, \tilde{h})$, where $i d_{N}:(N, h) \rightarrow(N, \tilde{h})$ is the identity map. Then we have

$$
\tau(\tilde{\pi})=\tau\left(i d_{N}\right) \text { and } \tau_{2}(\tilde{\pi})=\tau_{2}\left(i d_{N}\right),
$$

where $\tau(\phi)$ and $\tau_{2}(\phi)$ denote tension field and bitension field of a map $\phi$.

This theorem implies that the study of biharmonicity of a harmonic Riemannian submersion $\pi:(M, g) \rightarrow(N, h)$ reduces to the study of biharmonicity of the identity map $i d_{N}:(N, h) \rightarrow(N, \tilde{h})$.

\section{Gauss and Identity Maps}

Let $Q_{m-n}$ be the Grassmann manifold consisting of oriented linear $n$-subspaces of the Euclidean $m$-space $\left(\mathbb{E}^{m}, \bar{g}_{0}\right)$ equipped with the standard Euclidean metric $\bar{g}_{0}$. Then $Q_{m-n}$ admits a standard Riemannian metric $\widetilde{G}_{0}$ which makes $Q_{m-n}$ a compact symmetric space. In particular, $Q_{m-2}$ with a natural complex structure is holomorphically isometric to the complex quadric of complex dimension $m-2$. 


\subsection{Gauss Images}

Consider an immersion $\phi: M^{n} \rightarrow\left(\mathbb{E}^{m}, \bar{g}_{0}\right)$ from an $n$-manifold $M^{n}$ into a Euclidean $m$-space $\mathbb{E}^{m}$. Let $g_{0}$ denote the induced metric on $M^{n}$ via $\phi: M^{n} \rightarrow\left(\mathbb{E}^{m}, \bar{g}_{0}\right)$. The Gauss map $v: M^{n} \rightarrow\left(Q_{m-n}, \widetilde{G}_{0}\right)$ is the map obtained by parallel displacement of the tangent plane $d \phi\left(T_{p} M^{n}\right)\left(p \in M^{n}\right)$ in $\mathbb{E}^{m}$. In this section, we always assume that the Gauss map $v$ is a regular map so that the Gauss map $v$ induces a metric $G_{0}$ on $M^{n}$. In this way, $M^{n}$ admits two metrics $g_{0}$ and $G_{0}$ induced via $\phi$ and $\nu$, respectively.

Definition 7. A submanifold $M^{n}$ of $\mathbb{E}^{m}$ is said to have totally geodesic Gauss image if geodesics of $\left(M, G_{0}\right)$ are carried to geodesics of $\left(Q_{m-n}, \widetilde{G}_{0}\right)$ by the Gauss map.

The following result was proved by the author and Yamaguchi.

Theorem 35. $[67,68]$ A Euclidean submanifold $M^{n}$ in $\mathbb{E}^{m}$ has totally geodesic Gauss image if and only if the second fundamental form $B$ of $M^{n}$ in $\mathbb{E}^{m}$ satisfies

$$
\left(\bar{\nabla}_{X} B\right)(Y, Z)=B\left(\nabla_{X}^{G_{0}} Y-\nabla_{X} Y, Z\right)
$$

for vector fields $X, Y, Z$ tangent to $M^{n}$, where the Gauss image $\nabla^{G_{0}}$ is the Levi-Civita connection of $\left(M^{n}, G_{0}\right)$.

\subsection{Geometry of Gauss Transformations}

In this subsection, we discuss the identity map $i d_{M}:\left(M, G_{0}\right) \rightarrow\left(M, g_{0}\right)$ associated with an isometric immersion $\phi:\left(M, g_{0}\right) \rightarrow \mathbb{E}^{m}$. We call this identity map the Gauss transformation of $\phi$. More precisely, we present results for a surface in $\mathbb{E}^{m}$ to have harmonic, conformal, homothetic, or affine Gauss transformation. Here the identity map $i d_{M}:\left(M, G_{0}\right) \rightarrow\left(M, g_{0}\right)$ is called affine if it carries geodesics into geodesics.

Theorem 36. [2] An isometric immersion $\phi:\left(M, g_{0}\right) \rightarrow\left(\mathbb{E}^{m}, \bar{g}_{0}\right)$ of a surface is harmonic if and only if $\phi:\left(M, G_{0}\right) \rightarrow\left(\mathbb{E}^{m}, \bar{g}_{0}\right)$ is harmonic.

Theorem 36 implies the following.

Corollary 11. [2] Let $\phi: M \rightarrow\left(\mathbb{E}^{m}, \bar{g}_{0}\right)$ be an immersion of a surface $M$ into $\mathbb{E}^{m}$. Then the Gauss map $v:\left(M, G_{0}\right) \rightarrow\left(Q_{m-2}, \widetilde{G}_{0}\right)$ is harmonic and the identity map id $M:\left(M, G_{0}\right) \rightarrow\left(M, g_{0}\right)$ is homothetic if and only if

(1) $\left(M, g_{0}\right)$ has constant Gauss curvature and

(2) there exists a hypersphere $S^{m-1}$ of $\mathbb{E}^{m}$ such that $\phi:\left(M, g_{0}\right) \rightarrow S^{m-1}$ is harmonic.

Remark 9. Theorem 36 is false in general if $\operatorname{dim} M \geq 3$. In fact, for $n \geq 3$, there exists a hypersurface in $\mathbb{E}^{n+1}$ such that $\phi:\left(M, g_{0}\right) \rightarrow\left(\mathbb{E}^{n+1}, \bar{g}_{0}\right)$ is harmonic, but $\phi:\left(M, G_{0}\right) \rightarrow\left(\mathbb{E}^{n+1}, \bar{g}_{0}\right)$ is not harmonic.

Theorem 37. [2] Let $\phi: M \rightarrow\left(\mathbb{E}^{m}, \bar{g}_{0}\right)$ be an immersion of a surface $M$ into $\mathbb{E}^{m}$. Then the identity map $i d_{M}:\left(M, G_{0}\right) \rightarrow\left(M, g_{0}\right)$ is conformal and the Gauss map $v:\left(M, G_{0}\right) \rightarrow\left(Q_{m-2}, \widetilde{G}_{0}\right)$ is harmonic if and only if either

(a) $\phi:\left(M, g_{0}\right) \rightarrow\left(\mathbb{E}^{n+1}, \bar{g}_{0}\right)$ is harmonic, or

(b) there exists a hypersphere $S^{m-1}$ of $\mathbb{E}^{m}$ such that $\phi:\left(M, g_{0}\right) \rightarrow S^{m-1}$ is harmonic.

Theorem 38. [2] Let $\phi: M \rightarrow\left(\mathbb{E}^{m}, \bar{g}_{0}\right)$ be an immersion of a surface $M$ into $\mathbb{E}^{m}$. If id ${ }_{M}:\left(M, G_{0}\right) \rightarrow\left(M, g_{0}\right)$ is affine, then either $i d_{M}:\left(M, G_{0}\right) \rightarrow\left(M, g_{0}\right)$ is homothetic or both $(M, g)$ and $(M, G)$ are flat surfaces.

Theorem 39. [2] Let $\phi: M \rightarrow\left(\mathbb{E}^{m}, \bar{g}_{0}\right)$ be an immersion of a surface $M$ into $\mathbb{E}^{m}$. Then $i d_{M}:\left(M, G_{0}\right) \rightarrow$ $\left(M, g_{0}\right)$ is affine and the Gauss map $v:\left(M, G_{0}\right) \rightarrow\left(Q_{m-2}, \widetilde{G}_{0}\right)$ is harmonic if and only if $\left(M, g_{0}\right)$ has constant 
Gauss curvature and either (1) $\left(M, g_{0}\right)$ is immersed in a hypersphere of $\mathbb{E}^{m}$ as a minimal surface via $\phi$, or (2) $\left(M, g_{0}\right)$ is immersed as an open portion of the product surface of two planar circles via $\phi$.

\section{Laplace and Identity Maps}

Consider an isometric immersion $\phi:\left(M, g_{0}\right) \rightarrow\left(\mathbb{E}^{m}, \bar{g}_{0}\right)$ of a Riemannian $n$-manifold $M$ into a Euclidean $m$-space $\mathbb{E}^{m}$. Then the Laplace operator $\Delta$ of $\left(M, g_{0}\right)$ gives rise to a differentiable map

$$
L_{\phi}: M \rightarrow\left(\mathbb{E}^{m}, \bar{g}_{0}\right) ; p \mapsto(\Delta \phi)(p),
$$

called the Laplace map, where $\phi$ is regarded as the immersion as well as the position vector field of $M$ in $\mathbb{E}^{m}$. The image $L_{\phi}(M)$ of $M$ under map $L_{\phi}$ is called the Laplace image of $M$.

In this section, we assume that the Laplace map $L_{\phi}$ is a regular map, so that the Laplace image $L_{\phi}(M)$ admits an induced metric $g_{L_{\phi}}$ via $L_{\phi}$. In this way, $M$ admits two metrics $g_{0}$ and $g_{L_{\phi}}$. Hence, we may consider the differential geometry of the identity map $i d_{M}:\left(M, g_{0}\right) \rightarrow\left(M, g_{L_{\phi}}\right)$. Note that the identity map $i d_{M}:\left(M, g_{0}\right) \rightarrow\left(M, g_{L_{\phi}}\right)$ was called as the Laplace transformation in the book [7] of the author and Verstraelen.

The following question was asked and initially studied in [7].

Question 2. To what extent does the identity map $i d_{M}:\left(M, g_{0}\right) \rightarrow\left(M, g_{L_{\phi}}\right)$ determine the immersion $\phi:\left(M, g_{0}\right) \rightarrow\left(\mathbb{E}^{m}, \bar{g}_{0}\right) ?$

In this section we present some answers for this question from [7].

\subsection{Submanifolds with Homothetic Laplace Transformations}

Let $M=G / H$ be a homogeneous Riemannian manifold and $I(M)$ be the group of isometries on $M$. Then an isometric immersion $\psi: M \rightarrow M$ is said to be $G$-equivariant if there exists a homomorphism $\zeta: G \rightarrow I(M)$ such that $f(g(p))=\zeta(g) f(p)$ for each $g \in G$ and $p \in M$.

Theorem 40. Let $\phi:\left(M, g_{0}\right) \rightarrow\left(\mathbb{E}^{m}, \bar{g}_{0}\right)$ be an equivariant isometric immersion of a compact irreducible Riemannian homogeneous manifold into $\mathbb{E}^{m}$. Then the identity map $i d_{M}:\left(M, g_{0}\right) \rightarrow\left(M, g_{L_{\phi}}\right)$ is homothetic.

For an isometric immersion of $(M, g)$ into a Euclidean $m$-space $\mathbb{E}^{m}$ with homothetic identity map $i d_{M}:\left(M, g_{0}\right) \rightarrow\left(M, g_{L_{\phi}}\right)$, we have the following results from [7].

Theorem 41. Let $\phi:\left(M, g_{0}\right) \rightarrow\left(\mathbb{E}^{m}, \bar{g}_{0}\right)$ be an isometric immersion. If id ${ }_{M}:\left(M, g_{0}\right) \rightarrow\left(M, g_{L_{\phi}}\right)$ is homothetic, then we have:

(1) If $\phi$ is of finite type, the Laplace map $L_{\phi}: M \rightarrow \mathbb{E}^{m}$ is of finite type.

(2) If $M$ is compact, then the Laplace map $L_{\phi}$ is of $k$-type if and only if the immersion $\phi$ is of $k$-type.

(3) If $\phi$ is of finite type, then the Laplace map is of non-null finite type; in particular, if $x$ is of non-null k-type, then $L_{\phi}$ is of non-null $k$-type; and if $\phi$ is of null $k$-type, then $L_{\phi}$ is of non-null $(k-1)$-type.

Theorem 42. Let $\phi:\left(M, g_{0}\right) \rightarrow\left(\mathbb{E}^{m}, \bar{g}_{0}\right)$ be an isometric immersion. If id $M:\left(M, g_{0}\right) \rightarrow\left(M, g_{L_{\phi}}\right)$ is homothetic, then the Laplace image lies in a hypersphere of $\mathbb{E}^{m}$ as a minimal submanifold if and only if either (1) $M$ is a minimal submanifold of a hypersphere of $\mathbb{E}^{m}$ or $(2) \phi$ is of null 2-type.

Theorem 43. If $\phi:\left(M, g_{0}\right) \rightarrow\left(\mathbb{E}^{m}, \bar{g}_{0}\right)$ is an isometric immersion with parallel mean curvature vector, then $i d_{M}:\left(M, g_{0}\right) \rightarrow\left(M, g_{L_{\phi}}\right)$ is homothetic if and only if $M$ is immersed as a minimal submanifold of a hypersphere of $\mathbb{E}^{m}$ via $\phi$.

Theorem 44. Let $\phi:\left(M^{n}, g_{0}\right) \rightarrow\left(\mathbb{E}^{n+1}, \bar{g}_{0}\right)$ be a hypersurface of $\mathbb{E}^{n+1}$ with $n=2$ or 3 . Then id $M^{n}$ : $\left(M^{n}, g_{0}\right) \rightarrow\left(M^{n}, g_{L_{\phi}}\right)$ is homothetic if and only if $M^{n}$ is an open part of a hypersphere in $\mathbb{E}^{n+1}$ via $\phi$. 
Theorem 45. Let $\phi:\left(M^{2}, g_{0}\right) \rightarrow\left(\mathbb{E}^{4}, \bar{g}_{0}\right)$ be an isometric immersion with constant mean curvature. Then id $M^{2}:\left(M^{2}, g_{0}\right) \rightarrow\left(M^{2}, g_{L_{\phi}}\right)$ is homothetic if and only if $M^{2}$ is a minimal surface in a hypersphere of $\mathbb{E}^{4}$ via $\phi$.

\subsection{Geometry of Conformal or Harmonic Laplace Transformations}

Theorem 46. Let $\phi:\left(M^{n}, g_{0}\right) \rightarrow\left(\mathbb{E}^{n+1}, \bar{g}_{0}\right)$ be a hypersurface with $n \geq 4$. If the identity map id $M^{n}$ : $\left(M^{n}, g_{0}\right) \rightarrow\left(M^{n}, g_{L_{\phi}}\right)$ is conformal and $\left(M^{n}, g\right)$ has positive semi-definite Ricci tensor, then $\left(M^{n}, g\right)$ is conformally flat.

Theorem 47. Let $\phi:\left(M^{2}, g_{0}\right) \rightarrow\left(\mathbb{E}^{m}, \bar{g}_{0}\right)$ be an isometric immersion of a surface $M^{2}$ into $\mathbb{E}^{m}$. If id $M^{2}$ : $\left(M^{2}, g_{0}\right) \rightarrow\left(M^{2}, g_{L_{\phi}}\right)$ is conformal, then the Laplace image $L_{\phi}(M)$ is a minimal surface of $\mathbb{E}^{m}$ via $L_{\phi}$ if and only if $\phi$ is biharmonic.

Theorem 48. Let $\phi:\left(M, g_{0}\right) \rightarrow\left(\mathbb{E}^{m}, \bar{g}_{0}\right)$ be an isometric immersion with parallel mean curvature vector. Then the identity map id $M:\left(M, g_{0}\right) \rightarrow\left(M, g_{L_{\phi}}\right)$ is harmonic.

\subsection{Geometry of Laplace-Gauss Identity Maps}

Suppose $\phi:\left(M^{n}, g_{0}\right) \rightarrow\left(\mathbb{E}^{m}, \bar{g}_{0}\right)$ is an isometric immersion from a Riemannian $n$-manifold $M^{n}$ into $\mathbb{E}^{m}$. For simplicity, we assume that the Gauss map and the Laplace map associated with $\phi$ are regular. Thus we have the metrics $G_{0}$ and $g_{L_{\phi}}$ on $M^{n}$ via Gauss and Laplace maps, respectively. Therefore, we may consider the identity map

$$
i_{L G_{\phi}}:\left(M^{n}, g_{L_{\phi}}\right) \rightarrow\left(M^{n}, G_{0}\right)
$$

between the Laplace and Gauss images. We call this map the Laplace-Gauss identity map, or the LG-identity map of $\phi$ for short.

The following results on LG-identity maps are also obtained in [7].

Theorem 49. Let $\phi:\left(M, g_{0}\right) \rightarrow\left(\mathbb{E}^{m}, \bar{g}_{0}\right)$ be an isometric immersion. Then the LG-identity map $i_{L G_{\phi}}:$ $\left(M, g_{L_{\phi}}\right) \rightarrow\left(M, G_{0}\right)$ is conformal if and only if locally either $\phi$ has constant mean curvature function or $M$ is immersed as product of a planar curve and an affine $(n-1)$-space $\mathbb{E}^{n-1}$ via $\phi$.

Remark 10. A surface in $\mathbb{E}^{3}$ is called a surface of Delaunay if it has constant mean curvature function. Theorem 49 implies that every surface of Delaunay in $\mathbb{E}^{3}$ has homothetic LG-identity map.

Theorem 50. Let $\phi:\left(M^{n}, g_{0}\right) \rightarrow\left(\mathbb{E}^{n+1}, \bar{g}_{0}\right)$ be a hypersurface. Then the LG-identity map $i_{L G_{\phi}}:\left(M, g_{L_{\phi}}\right) \rightarrow$ $\left(M, G_{0}\right)$ is homothetic if and only if locally either $M$ has constant mean curvature function in $\mathbb{E}^{n+1}$ or $M$ is the product of an affine $(n-1)$-space $\mathbb{E}^{n-1}$ with a planar curve whose curvature function is given by

$$
\kappa(s)=\frac{n}{2}\left(\sqrt{a^{2} e^{-s^{2}}+4\left(c+c^{2}\right)}-a e^{-s}\right)
$$

for some positive constants a,c.

Theorem 51. Let $\phi: M^{n} \rightarrow S^{n+1} \subset \mathbb{E}^{n+2}$ be a hypersurface of a hypersphere $S^{n+1}$ of $\mathbb{E}^{n+2}$. Then $M$ has constant mean curvature function and $i_{L G_{\phi}}:\left(M, g_{L_{\phi}}\right) \rightarrow\left(M, G_{0}\right)$ is homothetic if and only if either

(1) $M^{n}$ is an open part of the product of two spheres $M^{k}(a) \times M^{n-k}(b)$ with some suitable radii a and $b$, or

(2) $M^{n}$ is an open part of a hypersphere of $S^{n+1}$.

Theorem 52. Let $\phi: M^{n} \rightarrow S^{n+1} \subset \mathbb{E}^{n+2}$ be a hypersurface of a hypersphere $S^{n+1}$ of $\mathbb{E}^{n+2}$. If $M$ has non-constant mean curvature function and $i_{L G_{\phi}}:\left(M, g_{L_{\phi}}\right) \rightarrow\left(M, G_{0}\right)$ is conformal, then 
(1) The gradient of the mean curvature function is an eigenvector of the shape operator $A$ of $M^{n}$ in $S^{n+1}$.

(2) The shape operator A has at most three distinct eigenvalues.

(3) The eigenvalue corresponding to the eigenvector given by the gradient of the mean curvature function is of multiplicity one.

Theorem 53. Let $\phi: M^{2} \rightarrow S^{3} \subset \mathbb{E}^{4}$ be an isometric immersion. Then $M^{2}$ has constant mean curvature in $S^{3}$ and the LG-identity map $i_{L G_{\phi}}:\left(M^{2}, g_{L_{\phi}}\right) \rightarrow\left(M^{2}, G_{0}\right)$ is conformal if and only if $M^{2}$ is either a totally umbilical surface or a minimal surface in $S^{3}$.

Theorem 54. Let $\phi: M^{2} \rightarrow S^{3} \subset \mathbb{E}^{4}$ be an isometric immersion. Then $M^{2}$ has constant Gauss curvature and $i_{L G_{\phi}}:\left(M^{2}, g_{L_{\phi}}\right) \rightarrow\left(M^{2}, G_{0}\right)$ is homothetic if and only if locally $M^{2}$ is a totally umbilical surface or a Clifford torus in $S^{3}$.

For further results in this respect, see the book [7].

\section{Identity Maps of Tangent Bundles}

For general information on geometry of tangent bundles, we refer to [69].

\subsection{Tangent Bundles}

Let $(M, g)$ be a pseudo-Riemannian manifold with Levi-Civita connection $\nabla$. The tangent bundle TM of $M$ consists of pairs $(x, X)$, where $x$ is a point in $M$ and $X$ a tangent vector to $M$ at $x$. The map $\pi: T M \mapsto M:(x, X) \mapsto x$ is the natural projection.

The tangent space $T_{(x, X)} T M$ at a point $(x, X)$ in $T M$ is a direct sum of the vertical subspace $\mathcal{V}_{(x, X)}=\operatorname{ker}\left(\left.d \pi\right|_{(x, X)}\right)$ and the horizontal subspace $\mathcal{H}_{(x, X)}$ with respect to the Levi-Civita connection $\nabla$ of $M$ :

$$
T_{(x, X)} T M=\mathcal{H}_{(x, X)} \oplus \mathcal{V}_{(x, X)}
$$

For any vector $Y \in T_{x} M$, there exists a unique vector $Y^{h} \in \mathcal{H}_{(x, X)}$ at $(x, X) \in T M$ which is called the horizontal lift of $Y$ to the point $(x, X)$ such that $d \pi\left(Y^{h}\right)=Y$; and a unique vector $Y^{v} \in \mathcal{V}_{(x, X)}$ which is called the vertical lift of $Y$ to the point $(x, X)$ such that $Y^{v}(d f)=Y(f)$ for all $f \in \mathcal{F}(M)$. Hence, every vector $\bar{Y} \in T_{(x, X)} T M$ can be decomposed as

$$
\bar{Y}=Y_{1}^{h}+Y_{2}^{v}
$$

for uniquely determined vectors $Y_{1}, Y_{2} \in T_{x} M$. The horizontal lift (respectively, vertical lift) of a vector field $X$ on $M$ to TM is the vector field $X^{h}$ (respectively, the vector field $X^{v}$ ) on TM whose value at $(x, X)$ is the horizontal lift (respectively, vertical lift) of $X_{x}$ to $(x, X)$.

The tangent bundle TM of $(M, g)$ equips with a metric $g^{S}$ in a natural way; called the Sasaki metric, which depends only on the metric $g$ of the base manifold $M$. The Sasaki metric $g^{S}$ on $T M$ is defined by the relations:

$$
\left\{\begin{array}{l}
g^{S}\left(X^{h}, Y^{h}\right)=g(X, Y) \\
g^{S}\left(X^{h}, Y^{v}\right)=0 \\
g^{S}\left(X^{v}, Y^{v}\right)=g(X, Y)
\end{array}\right.
$$

for vector fields $X, Y$ on TM. Intuitively, the Sasaki metric $g^{S}$ is constructed in such a way that the vertical and horizontal sub-bundles are orthogonal and the bundle map $\pi:\left(T M, g^{S}\right) \mapsto(M, g)$ is a pseudo-Riemannian submersion. 
12.2. Identity Maps of Tangent Bundles with Lift-Complete Metrics

Let $(M, g)$ be a Riemannian $n$-manifold. Oniciuc defined in [70] a pseudo-Riemannian metric $g^{c}$ on $T M$, called the lift-complete of $g$, defined by the following relations:

$$
\left\{\begin{array}{l}
g^{c}\left(X^{v}, Y^{v}\right)=0 \\
g^{c}\left(X^{h}, Y^{v}\right)=g(X, Y) \\
g^{c}\left(X^{h}, Y^{h}\right)=g(X, Y)
\end{array}\right.
$$

Via a non-linear connection on the tangent bundle TM, Oniciuc defined on TM in [70] the tensor field $G$ of type $(0,2)$, called of lift-complete type, by

$$
\left\{\begin{array}{l}
G\left(X^{v}, Y^{v}\right)=0 \\
G\left(X^{h}, Y^{v}\right)=g(X, Y) \\
G\left(X^{h}, Y^{h}\right)=g(X, Y) .
\end{array}\right.
$$

Oniciuc proved the following.

Theorem 55. [70] We have:

(a) The identity map id ${ }_{T M}:(T M, G) \rightarrow\left(T M, g^{c}\right)$ is totally geodesic if and only if the projection $\pi$ : $(T M, G) \rightarrow(M, g)$ is totally geodesic.

(b) The identity map id ${ }_{T M}:(T M, G) \rightarrow\left(T M, g^{c}\right)$ is biharmonic, i.e., $g^{c}$ is biharmonic with respect to $G$.

For detailed results in this respect, see [70].

12.3. Identity Maps of Tangent Bundles with g-Natural Metrics

For a Riemannian manifold $M$, the Sasaki metric $g^{S}$ is only one possible choice inside a very large family of Riemannian metrics on the tangent bundle $T M$ of $M$, known as Riemannian $g$-natural metrics. Those metrics are constructed in a "natural" way from the Riemannian metric $g$ over $M$.

The introduction of $g$-natural metrics by Kowalski and Sekizawa in [71] converts the classification of second order natural transformations of Riemannian metrics on manifolds to that of metrics on tangent bundles.

The set of $g$-natural metrics which depend on six smooth functions from $\mathbb{R}^{+}$to $\mathbb{R}$ has been completely described by Abbassi and Serih in [72] as follows.

Theorem 56. Let $(M, g)$ be a Riemannian manifold and $G$ be a g-natural metric on $T M$. Then there are six smooth functions $\alpha_{i}, \beta_{i}: \mathbb{R}^{+} \rightarrow \mathbb{R}, i=1,2,3$, such that for every $u, X, Y \in T_{x} M$, we have

$$
\left\{\begin{array}{l}
G_{(x, u)}\left(X^{h}, Y^{h}\right)=\left(\alpha_{1}+\alpha_{3}\right)\left(r^{2}\right) g_{x}(X, Y)+\left(\beta_{1}+\beta_{3}\right)\left(r^{2}\right) g_{x}(X, u) g_{x}(Y, u), \\
G_{(x, u)}\left(X^{h}, Y^{v}\right)=\alpha_{2}\left(r^{2}\right) g_{x}(X, Y)+\beta_{2}\left(r^{2}\right) g_{x}(X, u) g_{x}(Y, u), \\
G_{(x, u)}\left(X^{v}, Y^{h}\right)=\alpha_{2}\left(r^{2}\right) g_{x}(X, Y)+\beta_{2}\left(r^{2}\right) g_{x}(X, u) g_{x}(Y, u), \\
G_{(x, u)}\left(X^{v}, Y^{v}\right)=\alpha_{1}\left(r^{2}\right) g_{x}(X, Y)+\beta_{1}\left(r^{2}\right) g_{x}(X, u) g_{x}(Y, u),
\end{array}\right.
$$

where $r^{2}=g_{x}(u, u)$. For $n=1$, the same holds with $\beta_{i}=0, i=1,2,3$.

To state the next two theorems, we put

$$
\begin{aligned}
& \phi_{i}(t)=\alpha_{i}(t)+t \beta_{i}(t), \\
& \alpha(t)=\alpha_{1}(t)\left(\alpha_{1}+\alpha_{3}\right)(t)-\alpha_{2}^{2}(t), \\
& \phi(t)=\phi_{1}(t)\left(\phi_{1}+\phi_{3}\right)(t)-\phi_{2}^{2}(t)
\end{aligned}
$$


for all $t \in \mathbb{R}^{+}$. The following two results were proved by Abbassi and Calvaruso in [73].

Theorem 57. Let $(M, g)$ be a Riemannian n-manifold and let $G$ be an arbitrary g-natural metric on TM. Then the identity map id $d_{T M}:\left(T M, g^{S}\right) \rightarrow(T M, G)$ is harmonic if and only if the equation

$$
\phi_{3}^{\prime}=(n-1)\left[\beta_{1}-\alpha_{1}^{\prime}-\left(\alpha_{1}+\alpha_{3}\right)^{\prime}\right]
$$

holds and either (1) the horizontal and vertical distributions are orthogonal with respect to $G$, or $(2)(M, g)$ is an Einstein manifold with Ric $(u)=c u$, and $2 \phi_{2}^{\prime}+(n-1) \beta_{2}=c \alpha_{2}$ holds.

Theorem 58. Let $(M, g)$ be a Riemannian manifold and $G$ an arbitrary g-natural metric on $T M$. Then $i d_{T M}$ : $(T M, G) \rightarrow\left(T M, g^{S}\right)$ is harmonic if and only if the following two conditions are satisfied:

$$
\begin{gathered}
0=\frac{1}{\phi}\left\{\phi_{2} \phi_{1}\left(\phi_{1}+\phi_{3}\right)^{\prime}+\phi_{2}\left(\phi+\phi_{3}\right) \phi_{1}^{\prime}-2 \phi_{1}\left(\phi_{1}+\phi_{3}\right) \phi_{2}^{\prime}\right\} \\
+\frac{n-1}{\alpha}\left\{\phi_{2} \alpha^{\prime}-\phi_{1}\left[\alpha_{2}\left(\beta_{1}+\beta_{3}\right)-\left(\alpha_{1}+\alpha_{3}\right) \beta_{2}\right]-\phi_{2}\left[\left(\alpha_{1}+\alpha_{3}\right) \beta_{1}-\alpha_{2} \beta_{2}\right]\right\},
\end{gathered}
$$

and

$$
\begin{gathered}
0=\frac{1}{\phi}\left\{\left(\phi_{2}^{2}-\phi\right)\left(\phi_{1}+\phi_{3}\right)^{\prime}+\left(\phi_{1}+\phi_{3}\right)^{2} \phi_{1}^{\prime}-2 \phi_{2}\left(\phi_{1}+\phi_{3}\right) \phi_{2}^{\prime}\right\} \\
+\frac{n-1}{\alpha}\left[-\left(\phi_{1}+\phi_{3}\right) \alpha^{\prime}+\phi_{2}\left[\alpha_{2}\left(\beta_{1}+\beta_{3}\right)-\left(\alpha_{1}+\alpha_{3}\right) \beta_{2}\right]+\left(\phi_{1}+\phi_{3}\right)\left[\left(\alpha_{1}+\alpha_{3}\right) \beta_{1}-\alpha_{2} \beta_{2}\right]\right\} .
\end{gathered}
$$

12.4. Harmonic Metrics on Non-Reductive Homogeneous Manifolds

Assume $g$ and $\hat{g}$ are $G$-invariant pseudo-Riemannian metrics on a non-reductive homogeneous 4-manifold $M^{4}=G / K$. Let $\hat{g}^{S}$ (respectively, $\hat{g}^{h}$ and $\hat{g}^{c}$ ) denote the Sasaki metric (respectively, the horizontal lift and the complete lift) of $\hat{g}$ on the tangent bundle $T M^{4}$. In addition, let $g^{S}$ (respectively, $g^{h}$ and $g^{c}$ ) denote the Sasaki metric (respectively, the horizontal lift and the complete lift) of $g$.

In [74] by Zaeim and Atashpeykar proved the following.

Theorem 59. Let $\left(\mathrm{M}^{4}, g\right)$ be a non-reductive homogeneous 4-manifold. Then the following four statements are equivalent:

(a) The identity map id $M_{M^{4}}:\left(M^{4}, g\right) \rightarrow\left(M^{4}, \hat{g}\right)$ is harmonic, i.e., the pseudo-Riemannian metric $\hat{g}$ on $M^{4}$ is harmonic with respect to the pseudo-Riemannian metric $g$.

(b) The identity map $i d_{T M^{4}}:\left(T M^{4}, g^{S}\right) \rightarrow\left(T M^{4}, \hat{g}^{S}\right)$ is harmonic.

(c) The identity map id $T M^{4}:\left(T M^{4}, g^{h}\right) \rightarrow\left(T M^{4}, \hat{g}^{h}\right)$ is harmonic.

(d) The identity map id $T M^{4}:\left(T M^{4}, g^{c}\right) \rightarrow\left(T M^{4}, \hat{g}^{C}\right)$ is harmonic.

Remark 11. A complete classification of non-reductive pseudo-Riemannian homogeneous spaces up to dimension four was obtained earlier in [75] by Fels and Renner.

\subsection{Identity Maps of Tangent Bundles of Walker 4-Spaces}

Bejan and Drctă-Romaniuc investigated the total space of the tangent bundle $T W^{4}$ of a Walker 4-manifold $\left(W^{4}, g, \mathcal{D}\right)$ and proved the following.

Theorem 60. [40] Let $\left(W^{4}, g, \mathcal{D}\right)$ be a Walker 4-manifold. Then the following three statements are equivalent:

(a) The identity map $i d_{W^{4}}:\left(W^{4}, g, \mathcal{D}\right) \rightarrow\left(W^{4}, \hat{g}, \mathcal{D}\right)$ is harmonic, i.e., a Walker metric $\hat{g}$ on $W^{4}$ is harmonic with respect to $g$.

(b) The Sasaki metric $\hat{g}^{S}$ of a Walker metric $\hat{g}$ is harmonic with respect to the Sasaki metric $g^{S}$ of the Walker metric $g$. 
(c) The horizontal lift $\hat{g}^{h}$ of a Walker metric $\hat{g}$ is harmonic with respect to the horizontal lift $g^{h}$ of the Walker metric $g$.

\subsection{Identity Maps of Gödel-Type Spacetimes}

The Gödel metric, introduced by Kurt Gödel in [76], is an exact solution of the Einstein field equations such that the stress-energy tensor, which contains two terms, the first term representing the matter density of a homogeneous distribution of swirling dust particles (dust solution), and the second associated with a nonzero cosmological constant. Gödel spacetimes have been deeply investigated in physics (cf. e.g., [77-79]).

Gödel-type 4D spacetimes can be described by the Lorentzian metrics

$$
g=[d t+H(r) d \varphi]^{2}-d r^{2}-P^{2}(r) d \varphi^{2}-d z^{2},
$$

where $t$ is the time variable and $(r, \varphi, z)$ for $r \geq 0, \varphi, z \in \mathbf{R}$ (undetermined for $r=0$ ). Further, $H(r)$ and $P(r)$ are arbitrary smooth functions on $M$ and $g$ is non-degenerate where $P(r) \neq 0$.

The following three theorems were proved by Zaeim, Jafari and Yaghoubi in [80].

Theorem 61. Let $g$ and $\hat{g}$ be Gödel-type metrics on a 4-manifold $M^{4}$ with the metric $g$ given by (77) and $\hat{g}$ given by

$$
\hat{g}=[d t+\hat{H}(r) d \varphi]^{2}-d r^{2}-\hat{P}^{2}(r) d \varphi^{2}-d z^{2}
$$

Then the identity map $i d_{M^{4}}:\left(M^{4}, g\right) \rightarrow\left(M^{4}, \hat{g}\right)$ is harmonic (equivalently, the metric tensor $\hat{g}$ is harmonic with respect to $g$ ) if and only if

$$
\hat{H}^{\prime}(r)(\hat{H}(r)-H(r))-\hat{P}(r) \hat{P}^{\prime}(r)+P(r) P^{\prime}(r)=0 .
$$

Theorem 62. Let $\left(M^{4}, g\right)$ be homogeneous Gödel-type spacetime. In this case, a metric tensor $\hat{g}$ is harmonic with respect to $g$ if and only if $g=\hat{g}$.

Theorem 63. Let $\left(M^{4}, g\right)$ be a Gödel-type spacetime with the metric $g$ given by (77) and let $\hat{g}$ be an arbitrary Gödel-type metric of Equation (78). Then the following four statements are equivalent:

(a) The identity map id $M^{4}:\left(M^{4}, g\right) \rightarrow\left(M^{4}, \hat{g}\right)$ is harmonic, i.e., $\hat{g}$ is harmonic with respect to $g$.

(b) The Sasaki metric $\hat{g}^{S}$ is harmonic with respect to the Sasaki metric $g^{S}$.

(c) The horizontal lift $\hat{g}^{h}$ is harmonic with respect to the horizontal lift $g^{h}$.

(d) The complete lift $\hat{g}^{c}$ is harmonic with respect to the complete lift $g^{c}$.

Acknowledgments: The author would like to express his many thanks to the reviewers for many nice suggestions to improve the presentation of this article.

Funding: This research received no external funding.

Conflicts of Interest: The author declares no conflict of interest.

\section{References}

1. Nagano, T. Stability of harmonic maps between symmetric spaces. In Harmonic Maps; Springer: Berlin, Germany, 1982.

2. Chen, B.-Y.; Nagano, T. Harmonic metrics, harmonic tensors, and Gauss maps. J. Math. Soc. Jpn. 1984, 36, 295-313. [CrossRef]

3. Kobayashi, S.; Nomizu, K. Foundations of Differential Geometry, Volume I; Wiley-Interscience: New York, NY, USA, 1963.

4. Weyl, H. Reine Infinitesimal geometrie. Math. Z. 1918, 26, 384-411. [CrossRef]

5. Cotton, É. Sur les variétés à trois dimensions. Ann. Fac. Sci. Toulouse Ser. II 1899, 1, 385-438. [CrossRef] 
6. Chen, B.-Y. Geometry of Submanifolds; Dover Publications, Inc.: Mineola, NY, USA, 2019.

7. Chen, B.-Y.; Verstraelen, L. Laplace Transformations of Submanifolds; Katholieke Universiteit: Leuven, Belgium, 1995.

8. Chen, B.-Y. Pseudo-Riemannian Geometry, S-Invariants and Applications; World Scientific: Hackensack, NJ, USA, 2011.

9. Chen, B.-Y. Total Mean Curvature and Submanifolds of Finite Type, 2nd ed.; World Scientific: Hackensack, NJ, USA, 2015.

10. Chen, B.-Y. Differential Geometry of Warped Product Manifolds and Submanifolds; World Scientific: Hackensack, NJ, USA, 2017.

11. Chen, B.-Y. Submanifolds with parallel mean curvature vector in Riemannian and indefinite space forms. Arab J. Math. Sci. 2010, 16, 1-46.

12. Chen, B.-Y. On total curvature of immersed manifolds IV. Spectrum and total mean curvature. Bull. Inst. Math. Acad. Sin. 1979, 7, 301-311.

13. Chen, B.-Y. On the total curvature of immersed manifolds VI. Submanifolds of finite type and their applications. Bull. Inst. Math. Acad. Sin. 1983, 11, 309-328.

14. Chen, B.-Y. On submanifolds of finite type. Soochow J. Math. 1983, 9, 17-33.

15. Chen, B.-Y. Total Mean Curvature and Submanifolds of Finite Type; World Scientific: Singapore, 1984.

16. Chen, B.-Y. A report of submanifolds of finite type. Soochow J. Math. 1996, 22, 117-337.

17. Chen, B.-Y. Some open problems and conjectures on submanifolds of finite type: Recent development. Tamkang J. Math. 2014, 45, 87-108. [CrossRef]

18. Chen, B.-Y.; Morvan, J.-M.; Nore, T. Energie, tension et order des applications a valeurs dans un espace euclidien. C. R. Acad. Sci. Paris 1985, 301, 123-126.

19. Chen, B.-Y.; Morvan, J.-M.; Nore, T. Energy, tension and finite type maps. Kodai Math. J. 1986, 9, $406-418$. [CrossRef]

20. Chen, B.-Y. Linearly independent, orthogonal, and equivariant immersions. Kodai Math. J. 1991, 14, $341-349$. [CrossRef]

21. Chen, B.-Y.; Petrovic, M. On spectral decomposition of immersions of finite type. Bull. Austral. Math. Soc. 1991, 44, 117-129. [CrossRef]

22. Eells, J.; Sampson, J.H. Harmonic mappings of Riemannian manifolds. Am. J. Math. 1964, 86, 109-160. [CrossRef]

23. Smith, R.T. The second variation formula for harmonic maps. Proc. Am. Math. Soc. 1975, 47, $229-236$. [CrossRef]

24. Leung, P.F. A note on stable harmonic maps. J. Lond. Math. Soc. 1984, 29, 380-384. [CrossRef]

25. Xin, Y.L. Some remarks on stable harmonic maps. Duke Math. J. 1980, 47, 609-613. [CrossRef]

26. Hawking, S.W.; Ellis, G.F.R. The Large Scale Structure of Space-Time; Cambridge Univ. Press: Cambridge, UK, 1973.

27. Graham, C.; Robin; Lee, J.M. Einstein metrics with prescribed conformal infinity on the ball. Adv. Math. 1991, 87, 186-225. [CrossRef]

28. Yano, K.; Bochner, S. Curvature and Betti Numbers; Annals of Math. Studies 32; Princeton Univ. Press: Princeton, NJ, USA, 1953.

29. Yano, K.; Nagano, T. On geodesic vector fields in a compact orientable Riemannian space. Comm. Math. Helv. 1961, 35, 55-64. [CrossRef]

30. Liu, H.L. Harmonic indefinite metrics, harmonic tensors and harmonic immersions. Northeast. Math. J. 1991, 7, 397-405.

31. Balmuş, A. Biharmonic properties and conformal changes. An. Ştiinţ. Univ. Al. I. Cuza Iaşi. Mat. (N. S.) 2004, 50, 361-372.

32. Bejan, C.-L.; Duggal, K.L. Global lightlike manifolds and harmonicity. Kodai Math. J. 2005, 28, $131-145$. [CrossRef]

33. Pyo, Y.-S.; Kim, H.W.; Park, J.-S. On stability of Einstein warped product manifolds. Honam Math. J. 2010, 32, 167-176. [CrossRef]

34. Eells, J.; Lemaire, L. A report on harmonic maps. Bull. Lond. Math. Soc. 1978, 10, 1-68. [CrossRef]

35. Eells, J.; Lemaire, L. Another report on harmonic maps. Bull. Lond. Math. Soc. 1988, 20, 385-524. [CrossRef] 
36. Watanabe, Y.; Dohira, R. Remarks on harmonic metrics, harmonic tensors and holomorphic vector fields in a Kähler manifold. Math. J. Toyama Univ. 1995, 18, 137-146.

37. Vîlcu, A.D.; Vîlcu, G.E. Some remarks on harmonic metrics on hyper-Kähler manifolds. JP J. Geom. Topol. 2007, 7, 397-403.

38. Brozos-Vázquez, M.; García-Río, E.; Gilkey, P.; Nikčević, S.; ázquez-Lorenzo, R.V. The geometry of Walker manifolds. In Synthesis Lectures on Mathematics and Statistics; Krantz, S.G., Ed.; Washington University: St. Louis, MO, USA, 2009.

39. Walker, A.G. Canonical form for a Riemannian space with parallel field of null planes. Quart. J. Math. 1950, 1, 69-79. [CrossRef]

40. Bejan, C.-L.; Druţă-Romaniuc, S.-L. Structures which are harmonic with respect to Walker metrics. Mediterr. J. Math. 2015, 12, 481-496. [CrossRef]

41. Yano, K.; Nagano, T. Les champs des vecteurs geodesiques sur les espaces symetriques. C. R. Acad. Sci. Paris 1961, 252, 504-505.

42. Fardoun, A.; Ratto, A. On the second variation of the identity map of a product manifold. Tohoku Math. J. 2000, 52, 235-249. [CrossRef]

43. Nagano, T. On the minimum eigenvalues of the Laplacians in Riemannian manifolds. Sei. Pap. Coll. Gen. Ed. Univ. Tokyo 1961, 11, 177-182.

44. Ohnita, Y. Stability of harmonic maps and standard minimal immersions. Tohoku Math. J. 1986, 38, $259-267$. [CrossRef]

45. Casimir, H.B.G. Über die Konstruktion einer zu den irreduziblen Darstellungen halbeinfacher kontinuierlicher Gruppen gehrigen Differentialgleichung. Proc. R. Acad. Amst. 1931, 38, 844-846.

46. Chen, B.-Y. A New Approach to Compact Symmetric Spaces and Applications: A Report on Joint Work with Professor T. Nagano; Katholieke Universiteit: Leuven, Belgium, 1987.

47. Chen, B.-Y.; Nagano, T. Totally geodesic submanifolds of symmetric spaces II. Duke Math. J. 1978, 45, 405-425. [CrossRef]

48. Chen, B.-Y.; Nagano, T. A Riemannian geometric invariant and its applications to a problem of Borel and Serre. Trans. Am. Math. Soc. 1988, 308, 273-297. [CrossRef]

49. Aloff, S.; Wallach, N.R. An infinite family of distinct 7-manifolds admitting positive curved Riemannian structures. Bull. Am. Math. Soc. 1975, 81, 93-97. [CrossRef]

50. Park, J.-S. Stability of the Identity Map of SU(3)/T(k, l). Tokyo J. Math. 1994, 17, 218-289. [CrossRef]

51. Tanno, S. Instability of spheres with deformed Riemannian metrics. Kodai Math. J. 1987, 10, $250-257$. [CrossRef]

52. Urakawa, H. On the least positive eigenvalue of the Laplacian for compact group manifolds. J. Math. Soc. Jpn. 1979, 31, 209-226. [CrossRef]

53. Tanno, S. The identity map as a harmonic map of a $(4 r+3)$-sphere with deformed metrics. Kodai Math. J. 1993, 16, 171-180. [CrossRef]

54. Urakawa, H. Stability of harmonic maps and eigenvalues of the Laplacian. Trans. Am. Math. Soc. 1987, 301, 557-589. [CrossRef]

55. Blair, D.E. Contact Manifolds in Riemannian Geometry; Lecture Notes in Mathematics; Springer: Berlin/Heidelberg, Germany, 1976; Volume 509.

56. Alegre, P.; Blair, D.E.; Carriazo, A. Generalized Sasakian space forms. Israel J. Math. 2004, 141, $157-183$. [CrossRef]

57. Rehman, N.A. Stability on generalized Sasakian space forms. Math. Rep. 2015, 17, 57-64.

58. Jiang, G.Y. 2-harmonic maps and their first and second variational formulas. Chin. Ann. Math. Ser. A 1986, 7, 389-402.

59. Dimitric, I. Quadric Representation and Submanifold of Finite Type. Ph.D. Thesis, Michigan State University, East Lansing, MI, USA, 1989.

60. Dimitric, I. Submanifolds of $\mathbb{E}^{m}$ with harmonic mean curvature vector. Bull. Inst. Math. Acad. Sin. 1992, 20,53-65.

61. Caddeo, R.; Montaldo, S.; Oniciuc, C. Biharmonic submanifolds in spheres. Israel J. Math. 2002, 130, 109-123. [CrossRef]

62. Baird, P.; Kamissoko, D. On constructing biharmonic maps and metrics. Ann. Global Anal. Geom. 2003, 23, 65-75. [CrossRef] 
63. Ou, Y.-L.; Chen, B.-Y. Biharmonic Submanifolds and Biharmonic Maps in Riemannian Geometry; World Scientific: Hackensack, NJ, USA, 2020.

64. Baird, P.; Ou, Y.-L. Biharmonic conformal maps in dimension four and equations of Yamabe-type. J. Geom. Anal. 2018, 28, 3892-3905. [CrossRef]

65. Gu, C.H. Conformally flat spaces and solutions to Yang-Mills equations. Phys. Rev. D 1980, 21, 970-971.

66. Baird, P.; Loubeau, E.; Oniciuc, C. Harmonic and biharmonic maps from surfaces. Contemp. Math. 2011, 542, 223-230.

67. Chen, B.-Y.; Yamaguchi, S. Classification of surfaces with totally geodesic Gauss image. Indiana Univ. Math. J. 1983, 32, 143-154. [CrossRef]

68. Chen, B.-Y.; Yamaguchi, S. Submanifolds with totally geodesic Gauss image. Geom. Dedicata 1984, 15, $313-322$. [CrossRef]

69. Yano, K.; Ishihara, S. Tangent and Cotangent Bundles; Marcel Dekker Inc.: New York, NY, USA, 1973.

70. Oniciuc, C. Pseudo-Riemannian metrics on tangent bundle and harmonic problems. Bull. Belg. Math. Soc. Simon Stevin 2000, 7, 443-454. [CrossRef]

71. Kowalski, O.; Sekizawa, M. Natural transformations of Riemannian metrics on manifolds to metrics on tangent bundles, A classification. Bull. Tokyo Gakugei Univ. 1988, 40, 1-29.

72. Abbassi, M.T.K.; Serih, M. On some hereditary properties of Riemannian gnatural metrics on tangent bundles of Riemannian manifolds. Differ. Geom. Appl. 2005, 22, 19-47. [CrossRef]

73. Abbassi, M.T.K.; Calvaruso, G. Harmonic maps having tangent bundles with g-natural metrics as source or target. Rend. Semin. Mat. Univ. Politec. Torino 2010, 68, 37-56.

74. Zaeim, A.; Atashpeykar, P. Harmonic metrics on four dimensional non-reductive homogeneous manifolds. Czechoslovak Math. J. 2018, 68, 475-490. [CrossRef]

75. Fels, M.E.; Renner, A.G. Non-reductive homogeneous pseudo-Riemannian manifolds of dimension four. Can. J. Math. 2006, 58, 282-311. [CrossRef]

76. Gödel, K. An example of a new type of cosmological solutions of Einsteins field equations of gravitation. Rev. Mod. Phys. 1949, 21, 447-450. [CrossRef]

77. Gusin, P. Causal transformation of Gödel-type spacetimes in conformal field theory. Modern Phys. Lett. A 2010, 25, 2873-2884. [CrossRef]

78. Meessen, P.; Ortín, T. Gödel spacetimes, abelian instantons, the graviphoton background and other flacuum solutions. Nuclear Phys. B 2004, 684, 235-263. [CrossRef]

79. Camci, U. Symmetries of geodesic motion in Gödel-type spacetimes. J. Cosmol. Astropart. Phys. 2014, 7, 002. [CrossRef]

80. Zaeim, A.; Jafari, M.; Yaghoubi, M. Harmonic metrics on Gödel-type spacetimes. Int. J. Geom. Methods Mod. Phys. 2020, 17, 2050092. [CrossRef]

(C) 2020 by the author. Licensee MDPI, Basel, Switzerland. This article is an open access article distributed under the terms and conditions of the Creative Commons Attribution (CC BY) license (http://creativecommons.org/licenses/by/4.0/). 